\title{
Turbulent Mixing
}

\author{
Paul E. Dimotakis \\ Graduate Aeronautical Laboratories, California Institute of Technology, \\ Pasadena, California 91125; email: dimotakis@caltech.edu
}

Key Words turbulence, entrainment, scalar dispersion, diffusion, reacting flow

- Abstract The ability of turbulent flows to effectively mix entrained fluids to a molecular scale is a vital part of the dynamics of such flows, with wide-ranging consequences in nature and engineering. It is a considerable experimental, theoretical, modeling, and computational challenge to capture and represent turbulent mixing which, for high Reynolds number ( $R e$ ) flows, occurs across a spectrum of scales of considerable span. This consideration alone places high-Re mixing phenomena beyond the reach of direct simulation, especially in high Schmidt number fluids, such as water, in which species diffusion scales are one and a half orders of magnitude smaller than the smallest flow scales. The discussion below attempts to provide an overview of turbulent mixing; the attendant experimental, theoretical, and computational challenges; and suggests possible future directions for progress in this important field.

\section{INTRODUCTION}

Fluid entrained, or otherwise introduced in a turbulent region, is transported and dispersed across it by motions induced from the largest to the smallest eddies, where molecular diffusion has the opportunity to act, and where the ability of high Reynolds number turbulent flow to generate large interfacial surface area permits the otherwise slow molecular mixing to proceed effectively. Turbulent mixing can be viewed as a three-stage process (Eckart 1948) of entrainment, dispersion (or stirring), and diffusion, spanning the full spectrum of space-time scales of the flow. In liquids, where species (mass) diffusivities are much smaller than kinematic viscosities, it is useful to further split diffusive action into two steps, one in which viscosity acts (acquisition of small-scale vorticity) and the second where mass diffusion takes place (Batchelor 1959, Dimotakis 1986).

In the simplest case, mixing is passive, as occurs between passive scalars. This is labeled as Level-1 mixing. Examples are mixing of density-matched gases, the dispersion and mixing of nonreacting trace markers, such as pollutants, small temperature differences, small-particle smoke/clouds, or ink and other low-concentration dyes in a liquid, etc. Such mixing does not couple back on the flow dynamics; although dispersion and mixing are driven by the turbulent flow, a correct accounting of mixing is not required to describe the flow dynamics. 
Level-2 mixing is coupled to the dynamics, such as in mixing of differentdensity fluids in an acceleration/gravitational field, as in Rayleigh-Taylor (Rayleigh 1883, Taylor 1950) and Richtmyer-Meshkov (Richtmyer 1960, Meshkov 1969) instability flows, and mixing of the temperature and salinity fields in large-scale ocean currents and the thermohaline circulation (e.g., Adkins et al. 2002, Wunsch 2002, Wunsch \& Ferrari 2004), whose dynamics influence, if not dominate, climate and life on this planet. Mixing must be captured correctly to model such phenomena.

Level-3 mixing produces changes to the fluid(s), e.g., in composition, density, enthalpy conversion/release, pressure increase, etc., and is in turn coupled to the dynamics. Examples are the buoyancy-driven flow that both sustains and is driven by a candle, most combustion phenomena, detonations, and thermonuclear supernova explosions that are responsible for the production of all but the lightest elements in our universe (Colgate \& White 1966, Bethe \& Wilson 1985). An estimated 100 million supernova explosions in our galaxy have provided us with the oxygen we breathe and drink in our water, the calcium in our bones and statues, the carbon in all living cells, the silicon in sand and rocks, and the iron for our tools and cars (Burrows 2000). Turbulent mixing across the full spectrum of scales is a vital dynamic component in such explosions. Observations of light emission from nickel $\left({ }^{56} \mathrm{Ni}\right)$ in outer-region gases indicate transport by large-scale structures from inner regions, where ${ }^{56} \mathrm{Ni}$ is formed in the exploding star, to the outer shells (Janka 2002).

Progress in the study of turbulent mixing has mostly been confined to Level 1 in the hierarchy, with results for high Reynolds number flows mostly limited to a few canonical cases (e.g., grid/isotropic turbulence, channel and pipe flows, and free shear layers and jets) and largely based on empirical data. Theory, modeling, and even empirical knowledge of Level-2 and Level-3 mixing is less well developed and can fairly be characterized as an open research topic at this time, despite the crucial role such mixing plays.

The present discussion follows two recent reviews: Warhaft (2000), on passive scalar mixing, and Sawford (2001) on classical foundations of turbulent scalar dispersion, the kinematics of particle pairs, and two-point modeling closures. They should be consulted as companion references. The present review attempts to complement these, extending the discussion to more-complicated flows and mixing environments, including those at Levels 2 and 3 outlined above. A complete discussion would also cover mixing in compressible turbulent flows, an area important in its own right, with applications in many astrophysical and other contexts. However, a discussion of this topic is best handled separately and is not part of this review.

\section{DISPERSION AND MIXING SCALES}

For fluid entrained at the largest scales of the flow, scalar dispersion and mixing is hosted on the full spectrum of scales encountered in the turbulence cascade. The outer scale of the flow, $\delta$, e.g., the local transverse extent of a turbulent region 
sustained by an imposed shearing velocity difference, $U$, defines the local (outer) Reynolds number of the flow,

$$
R e=\frac{\rho U \delta}{\mu}=\frac{U \delta}{v},
$$

where $\rho$ is a reference density, $\mu$ a reference viscosity, and $v \equiv \mu / \rho$ the kinematic viscosity. Appropriate $\rho$ and $\mu$ estimates for this expression may require suitable mixture rules and may depend on an average, or prevalent, mixture ratio, for example, if the fluids undergoing mixing are characterized by disparate properties.

For incompressible flows, i.e., for $M_{\mathrm{t}} \equiv u^{\prime} / a \lesssim 0.3-0.5$, with $u^{\prime}$ the rms velocity and $a$ the sound speed, classical turbulence theory provides useful scaling estimates. Below the outer scale, $\delta$, the next scale in the cascade is the Taylor (1935) microscale, defined in terms of the transverse velocity correlation function, $g(r)$, for statistically isotropic flow,

$$
\lambda_{\mathrm{T}} \equiv\left[-\frac{1}{2} g^{\prime \prime}(0)\right]^{-1 / 2}=\left[\frac{15 v}{\varepsilon}\right]^{1 / 2} u^{\prime} .
$$

In the second equality, $\varepsilon=\alpha U^{3} / \delta$ is the average kinetic energy dissipation rate per unit fluid mass (Taylor 1935) and $\alpha$ is a small, flow-dependent, dimensionless variable, accepted as an approximately $R e$-independent constant in high- $R e$ flow. Thus, $\lambda_{\mathrm{T}}$ can be viewed (first equality in Equation 2a) as the spatial (persistence) scale across which local velocity values may be treated as approximately invariant (e.g., Pope 2000). Using on-axis values in the far field of a turbulent jet, for example, one finds,

$$
\frac{\lambda_{\mathrm{T}}}{\delta}=C_{\mathrm{T}} R e^{-1 / 2}, \quad \text { where } \quad C_{\mathrm{T}} \approx 2.3,
$$

with $\delta=\delta_{\text {jet }}(x) \simeq 0.4\left(x-x_{0}\right)$ equal to the local far-field jet diameter and $x_{0}$ a virtual origin (Dimotakis 2000).

The Taylor microscale can be used to define the Taylor Reynolds number,

$$
R e_{\mathrm{T}}=\frac{u^{\prime} \lambda_{\mathrm{T}}}{v} \propto R e^{1 / 2},
$$

with a (weakly) flow-dependent, order-unity proportionality constant. $R e_{\mathrm{T}}$ is useful where an outer-flow Reynolds number (Equation 1) is not appropriate, such as in grid-generated turbulence. Finally, as noted by H. Liepmann (private communication), the Taylor microscale can also be understood as the internal viscous shear-layer thickness associated with a large-scale motion spanning the full transverse extent, $\delta$, of the flow (e.g., Equation 2b). As such, it is the smallest scale generated by a $\delta$-size eddy/sweep and defines the range of scales directly connected to outer-scale dynamics by viscous action.

Following the K41 proposals (Kolmogorov 1941), the smallest velocity scale is given by (cf. also, Tennekes \& Lumley 1972, Pope 2000), 


$$
\lambda_{\mathrm{K}} \equiv\left(\nu^{3} / \varepsilon\right)^{1 / 4} \simeq C_{\mathrm{K}} \delta R e^{-3 / 4} .
$$

The constant of proportionality, $C_{\mathrm{K}}$, is (weakly) flow dependent and of order unity. The end of the $-5 / 3$ power-law regime in velocity spectra indicates that inner viscous scales, $\lambda_{v}$, are somewhat larger, with $\lambda_{v} \approx 50 \lambda_{\mathrm{K}}$ (Dimotakis 2000). This scale hierarchy provides important criteria for mixing, as discussed below.

In the context of turbulent diffusion and the smallest scalar-field scales, of interest is the Batchelor (1959) scale,

$$
\lambda_{\mathrm{B}} \simeq C_{\mathrm{B}} \lambda_{\mathrm{K}} S c^{-1 / 2} \simeq C_{\mathrm{B}} C_{\mathrm{K}} \delta R e^{-3 / 4} S c^{-1 / 2},
$$

defined here for Schmidt numbers, $S c \equiv v / \mathcal{D}>1$, where $\mathcal{D}$ is the species diffusivity. $C_{\mathrm{B}}$ is also of order unity (Batchelor 1959). These arguments (Equation 4) suggest a scalar diffusion scale, $\lambda_{\mathcal{D}}$, based on the viscous scale, i.e.,

$$
\lambda_{\mathcal{D}} \approx \lambda_{v} S c^{-1 / 2},
$$

analogous to the (physical) viscous scale versus the (defined) K41 scale (Dimotakis 2000).

Diffusion and viscous scales are comparable in gas-phase mixing $\left(S c_{\text {gas }} \approx 1\right)$. Schmidt numbers for liquids are significantly higher $\left(S c_{\text {liq }} \approx 600-3000\right)$, however, producing smaller scalar diffusion scales, $\left.1 / 50 \lesssim\left(\lambda_{\mathcal{D}} / \lambda_{\nu}\right)\right)_{\text {liq }} \lesssim 1 / 25$. Effective diffusivities can also be assigned for smoke, small-particle suspensions, clouds, etc., based on the Brownian motion of the particles. Resulting Schmidt numbers are high $\left(S c_{\text {particles }} \sim 10^{5}-10^{6}\right)$, result in very small $\lambda_{\mathcal{D}} / \lambda_{\nu}$ ratios, and are responsible, in part, for the sharp fronts common in particulate clouds.

These scaling relations illustrate the well-known difficulties in capturing passive turbulent mixing through Direct Numerical Simulation (DNS), i.e., no modeling. Resolving the diffusion scales requires a spatial dynamic range between $2 \delta / \lambda_{\mathcal{D}}$ and $2 \delta / \lambda_{B}$, and a number of grid points (or spectral degrees of freedom) given by $\left(2 \delta / \lambda_{\mathcal{D}}\right)^{3}$ and, quite possibly, as high as $\left(2 \delta / \lambda_{\mathrm{B}}\right)^{3}$. The number of time steps required to resolve inner scales during a single outer-flow time scale, $\delta / U$, adds a factor in the range of $\left(2 \delta / \lambda_{\mathcal{D}}\right)$ to $\left(2 \delta / \lambda_{\mathrm{B}}\right)$, for a computational burden that scales as $N_{\mathrm{LS}}\left(2 \delta / \lambda_{\mathcal{D}}\right)^{4} \propto N_{\mathrm{LS}} R e^{3} S c^{2}$, where $N_{\mathrm{LS}}$ is the number of outer (large-scale) times required for flow statistics to converge. Doubling $R e$ increases the burden by a factor of eight-nearly an order of magnitude. Capturing liquid-phase versus gas-phase mixing through DNS, at the same $R e$, requires a computational burden, roughly six orders of magnitude higher.

The burden on experiment is similar, both in terms of space-time resolution and volume of data that must be recorded, but with two important differences. Experimentally, one can focus the dynamic range recorded to the range of scales of interest, with some assurance that scales (and boundary conditions) not resolved are "solved" correctly by the flow. Secondly, if the interest is on a particular (scalar, or other) field alone, other fields (e.g., velocity, pressure, density, etc.) need not also be measured/recorded. They too are "solved" correctly by the flow. Such benefits are not bestowed on numerical simulations. 
Theoretically, experimentally, and computationally, matters are more complex in compressible turbulent flows $\left(M_{\mathrm{t}}>0.5\right)$, where regions of high dilatation and compression can occur, as well as shocks. These are responsible for additional dissipation mechanisms and dynamics that weaken the foundations of the Taylor (1935) and K41 theoretical framework. There is scant theoretical guidance in this flow regime, which is unfortunate, considering the rich physics it encompasses.

Chemical/biological/nuclear activity that may occur on mixing can introduce additional space/time scales, whose magnitude relative to the scales outlined above will depend on the ratio of the (Lagrangian) time, $\tau_{\text {mix }}$, required for mixing, to the time to complete chemical/other reactions, $\tau_{\text {react }}$, following mixing. Their relative magnitude is quantified by the Damkohler number,

$$
D a \equiv \frac{\tau_{\mathrm{mix}}}{\tau_{\text {react }}} .
$$

In a high- $D a$ environment, chemical-product formation is mixing-limited and provides a valuable diagnostic on molecular mixing, as discussed below.

\section{SPECIES TRANSPORT, DIFFUSION, AND MIXING}

Species transport and diffusion processes are described by a set of equations, one for each species $\alpha=1, \ldots, N$. Quite generally, one may write,

$$
\partial_{t}\left(\rho Y_{\alpha}\right)+\partial_{\mathbf{x}} \cdot\left[\rho Y_{\alpha}\left(\mathbf{u}+\mathbf{v}_{\alpha}\right)\right]=\varpi_{\alpha},
$$

where $\mathbf{u}$ is the local (mass-averaged) flow velocity, $\mathbf{v}_{\alpha}$ is the $\alpha$-species diffusion velocity in the $\mathbf{u}$ frame, and $\varpi_{\alpha}$ is the $\alpha$-species (chemical, nuclear, biological, etc.) production/consumption rate. Summing over species $\left(\sum_{\alpha} Y_{\alpha}=1\right.$ and $\sum_{\alpha} \varpi_{\alpha}=$ $0)$ yields $\sum_{\alpha} Y_{\alpha} \mathbf{v}_{\alpha}=0$ and $\partial_{t} \rho+\partial_{\mathbf{x}} \cdot(\rho \mathbf{u})=0$, as expected.

If species-concentration gradients contribute the dominant diffusive-flux component, we may write to lowest order, $\mathbf{v}_{\alpha}=-\mathcal{D}_{\alpha} \partial_{\mathbf{x}} \ln \left(Y_{\alpha}\right)$, where $\mathcal{D}_{\alpha}$ is the $\alpha$-species diffusivity. If, in addition, species are individually conserved $\left(\varpi_{\alpha}=0\right)$ and if fluid density is uniform, the transport equations simplify to the familiar (Fickian) species-conservation equations,

$$
\mathrm{D}_{t} Y_{\alpha}=\partial_{\mathbf{x}} \cdot\left(\mathcal{D}_{\alpha} \partial_{\mathbf{x}} Y_{\alpha}\right)
$$

where $\mathrm{D}_{t}$ is the Lagrangian derivative in the mass-averaged $\mathbf{u}$-frame.

For an ideal gas, $\left|\mathbf{v}_{\alpha}\right|_{\max } \sim \bar{c}_{\alpha} \ell_{\alpha} / \lambda_{\mathcal{D}}$, where $\bar{c}_{\alpha}$ is the mean relative (thermal) speed between the $\alpha$-species and its colliding partners, and $\ell_{\alpha}$ the mean free path of $\alpha$ molecules. In turn, we have $\bar{c}_{\alpha} \sim\left(k_{\mathrm{B}} T / m_{\alpha}\right)^{1 / 2} \sim a\left(\mathcal{M} / \mathcal{M}_{\alpha}\right)^{1 / 2}$, with $m_{\alpha}$ the $\alpha$-species molar (atomic/molecular) mass, $a$ and $\mathcal{M}$ the speed of sound and molar mass (molecular weight) of the gas mixture, and $\mathcal{M}_{\alpha}$ the $\alpha$-species molar mass. Within the Fickian-diffusion framework (Equation 8), differential-diffusion effects are then anticipated in mixtures of species with disparate collision cross-sections or molar masses. This is revisited below. 
If the mixing species are minor components in a common background diluent, the diffusivities, $\mathcal{D}_{\alpha}$, can be approximated by the binary-diffusion coefficients of the $\alpha$-species and the common background, and are thermodynamic functions of state. If, in addition, the local thermodynamic state is independent of the $Y_{\alpha}$, the transport-diffusion equations are linear in the $Y_{\alpha}$ fields. In that case and for a given (turbulent) velocity field, the solution may, at least formally, be regarded as a convolution of a Green's function over the space-time distribution of sources in the flow; a potentially useful construct in formulating statistical models of dispersion from point sources.

In this environment, turbulent mixing will yield $Y_{\alpha}(\mathbf{x}, t)$ values bounded by the min-max values in the initial/boundary conditions (the right-hand side of Equation 8 is a local spatial averaging operator). Equation 8 is also consistent with the notion of species transport and diffusion as a process that tends to homogenize fluid composition. However, Equation 8 is incomplete, as is seen by applying it to a gedanken experiment, in which helium and argon are jetted and mixed into a tall pressure vessel. After sufficient time, the flow will come to rest, i.e., $\mathrm{D}_{t} Y_{\alpha}=0$, under the action of viscosity. However, Equation 8 would then predict a final state of uniform helium-argon mixture profiles, missing the weak, in this case, density and compositional gravitational stratification.

Species diffusion is fundamentally driven by fluxes prescribed by the tendency toward (local) thermodynamic equilibrium and could be expressed as proceeding along components of the gradient of the local chemical potential (e.g., Landau \& Lifshitz 1997, Ch. 6). Diffusive transport need not lead to homogenization and can result in partial segregation (unmixing) of species, even if they are initially homogeneously mixed, as in the example above, or in a centrifuge. Chemical potential gradient components are proportional to species-concentration gradients, as in the Fickian diffusion approximation, but also possess components proportional to gradients of pressure, temperature, and any other variables whose differentials enter as chemical potential differentials, as in (differences in) body forces, as in an electrostatic precipitator, for example.

Species-diffusion velocities can be derived from the Boltzmann equation (Hirschfelder et al. 1954),

$$
\mathbf{v}_{\alpha}=-\sum_{\beta} D_{\alpha \beta} \mathbf{d}_{\beta}-\frac{\mathrm{D}_{\alpha}^{(T)}}{T} \partial_{\mathbf{x}} T
$$

where $D_{\alpha \beta}$ is the diffusion-coefficient matrix, $D_{\alpha}^{(T)}$ the $\alpha$-species thermal-diffusion coefficient, and

$$
\mathbf{d}_{\alpha} \equiv \partial_{\mathbf{x}} X_{\alpha}+\frac{1}{p}\left[\left(X_{\alpha}-Y_{\alpha}\right) \partial_{\mathbf{x}} p+\rho Y_{\alpha} \sum_{\beta} Y_{\beta}\left(\mathbf{f}_{\beta}-\mathbf{f}_{\alpha}\right)\right],
$$

with $X_{\alpha}$ and $\mathbf{f}_{\alpha}$ the $\alpha$-species mole fraction and body-force (acceleration) fields. However, species-diffusion velocities for nonideal-gas and condensed-phase mixtures are not as readily derivable from first principles. 
Non-Fickian terms in Equation 9 can be important in some turbulent-mixing environments, as in turbulent flames, for example, where a significant variance in species diffusivities can be encountered, and where large temperature gradients, e.g., $\left|\partial_{\mathbf{x}} T\right| \sim 10^{6} \mathrm{~K} / \mathrm{m}$, are not uncommon.

The pressure-gradient (barotropic) term can also be important in turbulent flows. Turbulent vortex cores are scaled by the inner viscous scale, $\lambda_{v}$ (cf. text following Equation 3), with circumferential velocities of the order of the turbulence rms velocities, $u^{\prime}$ (e.g., Jimenez et al. 1993, Jimenez \& Wray 1998). These are wellapproximated as $R e$-independent fractions of outer velocity scales, e.g., $u^{\prime} \approx$ $0.2 U$. The expected peak Lagrangian (centifugal) fluid acceleration can then be approximated by, $g_{\mathrm{f}} \approx u^{\prime 2} / \lambda_{v} \approx 5 \times 10^{-4}\left(U^{2} / \delta\right) R e^{3 / 4}$. For $U \simeq 100 \mathrm{~m} / \mathrm{s}, \delta \simeq$ $4 \mathrm{~cm}$, and $R e \simeq 10^{5}$, for example, we have, $g_{\mathrm{f}} \approx 1 \times 10^{6} \mathrm{~m} / \mathrm{s}^{2} \approx 1 \times 10^{5} g_{0}$, where $g_{0}$ is the acceleration of gravity. Such values are comparable to those encountered in high-performance centrifuges.

Equation 9 (or statistical mechanics) provides a scaling for local concentration excursions in inner-scale vortices, i.e.,

$$
\ln \left\langle\frac{X_{\alpha}\left(r=\lambda_{\nu}\right)}{X_{\alpha}(r=0)}\right\rangle \sim-\frac{m_{\alpha}}{k_{\mathrm{B}} T} \int_{0}^{\lambda_{\nu}} g_{\mathrm{f}} \mathrm{d} z \sim-\frac{m_{\alpha} u^{\prime 2}}{k_{\mathrm{B}} T} \sim-\frac{\gamma \mathcal{M}_{\alpha}}{\mathcal{M}} M_{\mathrm{t}}{ }^{2},
$$

where $\gamma$ is the specific-heat ratio. Disparate molar masses (large differences between $\mathcal{M}_{\alpha}$ and $\mathcal{M}$, and between $X_{\alpha}$ and $Y_{\alpha}$ ) can lead to instantaneous local segregation between premixed species in compressible turbulence (moderate to high $M_{\mathrm{t}}$ ). In this regime, local values for $Y_{\alpha}$ outside the min-max bounds in initial/boundary conditions are possible. The argument above, however, is only indicative and derives from incompressible-turbulence scaling, and may not be strictly applicable to compressible turbulence where it predicts these effects are expected.

Interactions with turbulence are more likely to lead to segregation in the case of particle/droplet clouds, an issue of importance to the dynamics of early cloud formation in the atmosphere (e.g., Shaw et al. 1998, Falkovich et al. 2002, Shaw 2003). Approximating the acceleration of an isolated particle in terms of $\dot{\mathbf{v}}_{\mathrm{p}}=$ $\left(\mathbf{u}-\mathbf{v}_{\mathrm{p}}\right) / \tau_{\mathrm{p}}+\mathbf{g}$, where $\mathbf{u}$ is the flow velocity, $\mathbf{v}_{\mathrm{p}}$ is the particle-cloud velocity, $\tau_{\mathrm{p}}$ is the particle response time, and $\mathbf{g}$ is the gravitational acceleration, we have $\mathbf{v}_{\mathrm{p}} \simeq \mathbf{u}+$ $(\mathbf{g}-\dot{\mathbf{u}}) \tau_{\mathrm{p}}+O\left(\tau_{\mathrm{p}}^{2}\right)$. Treating the particle number density, $n_{\mathrm{p}}(\mathbf{x}, t)$, as a continuum possessing its own velocity field, $\mathbf{v}_{\mathrm{p}}$, we have (Robinson 1956, Shaw 2003),

$$
\partial_{\mathbf{x}} \cdot \mathbf{v}_{\mathrm{p}} \simeq-\tau_{\mathrm{p}} \partial_{\mathbf{x}} \cdot\left(\mathbf{u} \cdot \partial_{\mathbf{x}} \mathbf{u}\right)=-\tau_{\mathrm{p}}\left(4 \mathbf{S}: \mathbf{S}-\omega^{2}\right),
$$

with $\mathbf{S}$ the strain-rate tensor and $\omega^{2}$ the entrophy. The consequences are also familiar to experimentalists who rely on particles as Lagrangian markers for various velocity-measuring techniques (Laser-Doppler Velocimetry, Particle-Imaging Velocimetry, etc.). For example, regions of intense vorticity (vortex cores) can be devoid of particles that centrifuge out $\left(\partial_{\mathbf{x}} \cdot \mathbf{v}_{\mathrm{p}}>0\right)$, leading to potentially serious conditional-sampling biases and systematic errors. 


\section{THE MIXING TRANSITION}

Many flows exhibit qualitatively different behavior beyond a transition Reynolds number,

$$
R e_{\text {tr }} \approx 1-2 \times 10^{4}, \quad \text { or, equivalently, } \quad R e_{\mathrm{Ttr}} \approx 10^{2}
$$

(Dimotakis 2000). This transition is encountered in gas- and liquid-phase shear layers, jets, pipe flow, boundary layers (with $R e$ based on boundary-layer displacement thickness), bluff-body wakes, grid turbulence and DNS of turbulence in a periodic box, Couette-Taylor flow, high Rayleigh number $(R a)$ thermal convection $\left(R e \sim R a^{1 / 2}\right.$ ), and Richtmyer-Meshkov instability flow, as well as in vortex rings (Glezer 1988, with $R e \equiv \Gamma / v$ ), transverse jets in a cross flow (Shan \& Dimotakis 2001), and, quite possibly, in all flows.

This transition reflects a change in the flow dynamics and manifests itself through a broader spectrum of eddying scales and a weaker Reynolds number dependence of various flow phenomena for higher Reynolds numbers yet, i.e., for $R e>R e_{\text {tr }}$, and often marks the beginning of a near $-5 / 3$ power-law regime in the energy spectrum with increasing $R e$. It is characterized by an increase in strain rates and attendant area of interfacial level sets across which mixing occurs and, as a consequence, an increase in mixing, hence the term.

Examples from liquid-phase shear layers and jets are shown in Figures 1 and 2 , respectively. From the flows listed above, we note that this transition occurs in both gas- and liquid-phase flows (not a Schmidt-number effect) as well as in wall-bounded and free-shear flows (independent of large-scale flow structure). Its
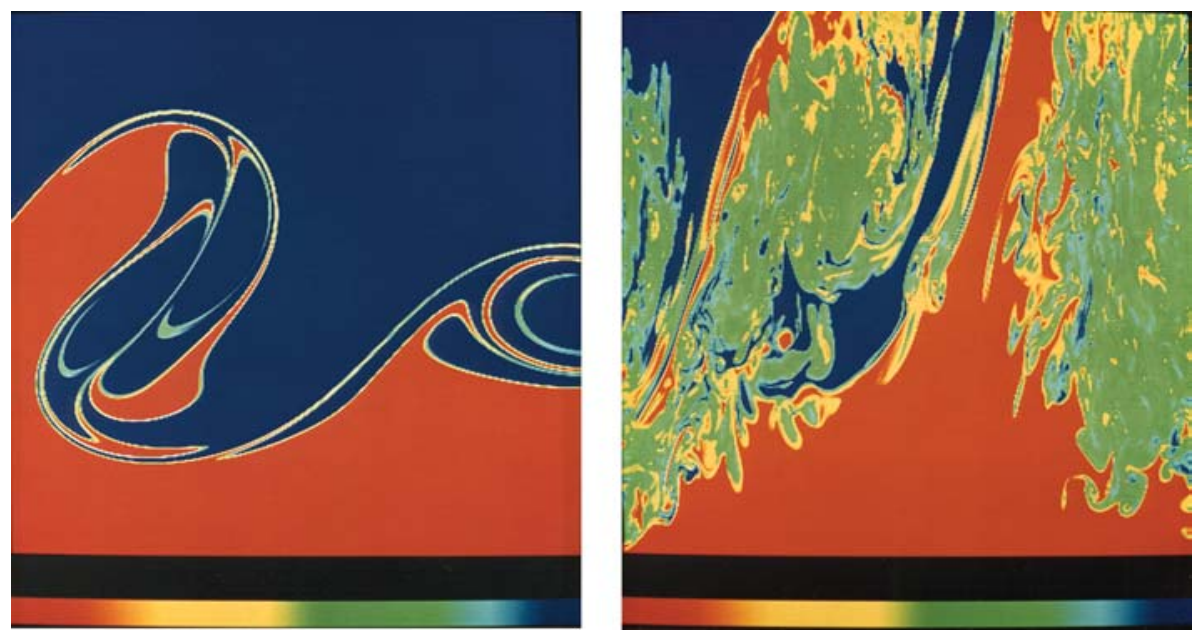

Figure 1 Liquid-phase shear-layer slices of passive scalar field. Color codes high-speed fluid mole fraction. Left: $R e_{\delta} \simeq 1.75 \times 10^{3}$, right: $R e_{\delta} \simeq 2.3 \times 10^{4}$ (Koochesfahani \& Dimotakis 1986). 

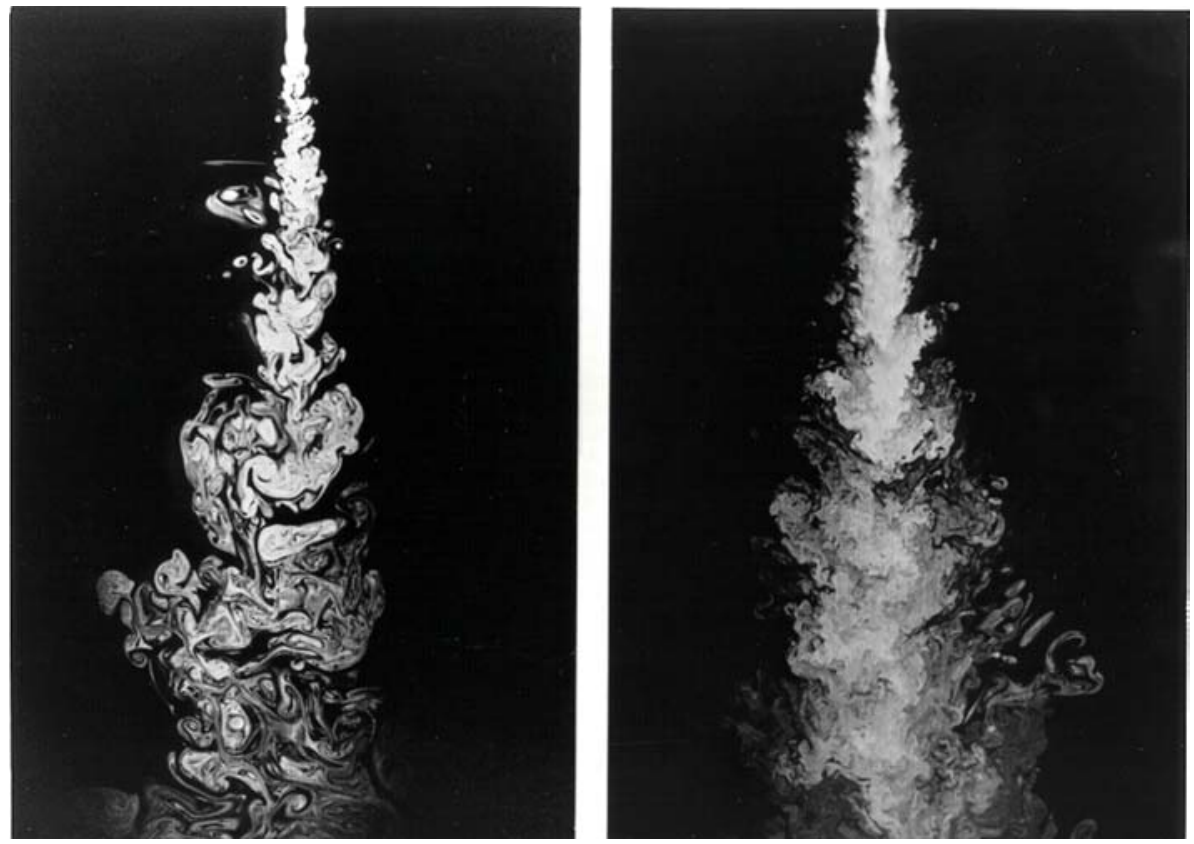

Figure 2 Liquid-phase turbulent-jet symmetry-plane slices of passive scalar field. Grey scale (contrast-enhanced) codes jet-fluid mole fraction. Left: $R e \simeq 2.5 \times 10^{3}$, right: $R e \simeq$ $1.0 \times 10^{4}$ (Dimotakis et al. 1983).

origins must then be sought in the dynamics over the internal range of scales, i.e., for scales $\lambda$, such that $\lambda_{\mathrm{K}} \ll \lambda \ll \delta$.

The transition coincides with when energy spectra start to osculate an approximately $-5 / 3$ logarithmic slope with increasing $R e$, that may, in turn, be identified with the emergence of an inertial inviscid range of eddies. This suggests that the post-transition regime requires a sufficient scale separation to support quasiinviscid dynamics. Expanding on the H. Liepmann idea on the similarity between the Taylor scale and the viscous-layer scale dependence on $R e$, we define the Liepmann-Taylor scale, $\lambda_{\mathrm{LT}}$, as a laminar scale,

$$
\lambda_{\mathrm{LT}}=5.0 \delta R e^{-1 / 2},
$$

where the numerical prefactor corresponds to the thickness of an internal laminar shear layer developing across the $\delta$ extent of the flow, as well as for a Blasius boundary layer (Dimotakis 2000). Because viscous effects span from the outer scale, $\delta$, to $\lambda_{\mathrm{LT}}$ and influence inner scales below $\lambda_{v} \approx 50 \lambda_{\mathrm{K}}$, inviscid dynamics require, at a minimum, room for $\lambda$-size eddies such that,

$$
\delta>\lambda_{\mathrm{LT}}>\lambda>\lambda_{v}>\lambda_{\mathrm{K}} \Rightarrow \frac{\lambda_{\mathrm{LT}}}{\lambda_{v}} \gtrsim 0.1 R e^{1 / 4} \gtrsim 1 \Rightarrow R e \gtrsim 10^{4},
$$

in accord with observation. 
Experimental evidence indicates that $R e$ effects on the dynamics and mixing become substantially weaker with increasing $R e$, for $R e>R e_{\text {tr }} \approx 10^{4}$. Conversely, experiment/DNS results for $R e \lesssim R e_{\text {tr }} \approx 10^{4}$ should be viewed with caution, with extrapolations from such results to the higher $R e$ 's typically of interest (fully developed turbulence) of dubious validity.

The discussion below focuses on mixing in high-Re (fully developed, postmixing-transition, $R e>R e_{\text {tr }}$ ) flows.

\section{LEVEL 1: PASSIVE-SCALAR MIXING}

Here, mixing and mixed fluid refer to molecularly mixed fluid, i.e., fluid whose local composition has been altered by interdiffusion of two, or more, fluids or species. Simplifying the discussion to mixing of two fluids for which a conserved scalar formulation is an adequate approximation (negligible differential-diffusion effects), the local composition can be described by the mixture-fraction field, $Z(\mathbf{x}, t)$, with values $\epsilon \leq Z \leq 1-\epsilon$ representing mixed fluid, for some small $\epsilon$. This permits the species mass-fraction fields, $Y_{\alpha}(\mathbf{x}, t)$, to be approximated as functions of the mixture-fraction field alone, i.e., $Y_{\alpha}(\mathbf{x}, t)=Y_{\alpha}(Z)=Y_{\alpha}[Z(\mathbf{x}, t)]$.

Statistics of the mixed-fluid mixture-fraction field can, at least in principle, be expressed in terms of the Probability Density Function (PDF) of composition, $p(Z ; \mathbf{x} ; R e, S c, \ldots)$. For flow developing along $x$ that is two-dimensional (2D) in the mean, the mixed-fluid (thickness) fraction can be expressed in terms of the PDF by excluding the probability of unmixed fluid, i.e. (with a weak dependence on $\epsilon$ ),

$$
\frac{\delta_{\mathrm{m}}(x)}{\delta(x)}=\frac{1}{\delta(x)} \int_{y} \int_{Z=\epsilon}^{1-\epsilon} p(Z ; x, y) \mathrm{d} Z \mathrm{~d} y .
$$

In practice, direct measurements of mixing in high-Re flows are difficult. DNS on ever more powerful computational resources recently permitted some valuable studies of mixing, even though the inexorable scaling considerations (section 2) limit those studies to moderate, or near-transitional, $R e$ 's and near-unity $S c$ 's. Probing the statistics of $Z(\mathbf{x}, t)$ by direct measurement in experiments, as necessary to compile $p(Z ; \mathbf{x})$ for Equation 15, for example, requires full resolution of turbulent diffusion space-time scales (section 2).

Under-resolved measurements do not distinguish between a homogeneous 50:50 mixture and 50:50 occupancy by unmixed fluids in the measurement time interval or probe volume, for example, and produce totally erroneous PDF estimates; a gas-phase $(S c \approx 1)$ shear layer with $\delta \simeq 3 \mathrm{~cm}$ and $\Delta U \approx 50 \mathrm{~m} / \mathrm{s}\left(R e \simeq 10^{5}\right)$, and $U_{\mathrm{c}} \approx\left(U_{1}+U_{2}\right) / 2 \approx \Delta U$, yields $\lambda_{\mathrm{K}} \approx \lambda_{\mathrm{B}} \approx 5 \mu \mathrm{m}$ and $t_{\mathrm{B}}=\lambda_{\mathrm{B}} / U_{\mathrm{c}} \approx 0.1 \mu \mathrm{s}$. Although viscous/diffusion scales are larger (cf. Equation 3 and discussion following), Nyquist and other sampling requirements (e.g., stemming from finite signal-to-noise considerations) render direct $Z(\mathbf{x}, t)$ measurements (e.g., with fine 
cold wires for temperature, or lasers for species concentration) beyond reach in high-Re flows for the foreseeable future. Direct measurements in high-Re liquidphase shear layers, $\left(\lambda_{\mathcal{D}}\right)_{\text {gas }} \approx 30\left(\lambda_{\mathcal{D}}\right)_{\text {liq }}$, are even further from the offing, if at all possible.

In view of this difficulty and the need to compare theory with experiment, the means by which mixing is actually measured in high-Re flows are important. Mixed-fluid fraction (Equation 15) and other mixing statistics can be estimated using irreversible chemical reactions in the fast-kinetic regime $(D a \gg 1$ in Equation 6). In this regime, the number of moles of chemical product formed-equivalently, the temperature rise from chemical-product formation in a fluid of uniform heat capacity - derives from the number of moles reacted and, in turn, from the number of reactant moles mixed on a molecular scale. The mean temperature rise $\Delta T\left(\mathbf{x} ; Z_{\phi}\right)=\left\langle\Delta T\left(\mathbf{x}, t ; Z_{\phi}\right)\right\rangle_{t}$, corresponding to an equivalence ratio, $\phi$ (moles of high-speed fluid required to completely consume a mole of low-speed fluid in a shear layer), or a stoichiometric-mixture fraction, $Z_{\phi}=\phi /(1+\phi)$, normalized by the adiabatic flame temperature rise of the reaction, $\Delta T_{\mathrm{f}}\left(Z_{\phi}\right)$, can be integrated across a turbulent 2D shear layer to yield the chemical product thickness (Dimotakis 1991),

$$
\begin{aligned}
\delta_{\mathrm{P}}\left(x ; Z_{\phi}\right) & \left.=\int_{y}\left[\int_{0}^{Z_{\phi}} \frac{Z}{Z_{\phi}}+\int_{Z_{\phi}}^{1} \frac{1-Z}{1-Z_{\phi}}\right\} p(Z ; x, y) \mathrm{d} Z\right] \mathrm{d} y \\
& =\int_{y} \frac{\Delta T\left(x, y ; Z_{\phi}\right)}{\Delta T_{\mathrm{f}}\left(Z_{\phi}\right)} \mathrm{d} y .
\end{aligned}
$$

The ratio $\delta_{\mathrm{P}}\left(Z_{\phi}\right) / \delta$ measures the volume (mole) fraction occupied by chemical product formed with entrained freestreams at a stoichiometric mixture ratio, $\phi$. Combining a low- $Z_{\phi}$ experiment with an experiment at $1-Z_{\phi}$ (runs at $\phi$ and $1 / \phi)$, we have,

$$
\frac{\delta_{\mathrm{m}}}{\delta} \simeq\left(1-Z_{\phi}\right)\left[\frac{\delta_{\mathrm{P}}\left(Z_{\phi}\right)}{\delta}+\frac{\delta_{\mathrm{P}}\left(1-Z_{\phi}\right)}{\delta}\right],
$$

with $\epsilon \approx Z_{\phi} / 2$ (Equation 15). This is quantitative, exploits a molecular probe to measure a molecular process, and obviates the need for high space-time resolution (only average quantities need to be measured).

To relate $\delta_{\mathrm{m}} / \delta$ to flow parameters, we note that entrainment is driven by largescale dynamics, dispersion by large and intermediate scale dynamics, and mixing by the small (viscous/diffusive) scales, that dominate, in turn, the surface-tovolume ratio, $\mathcal{S}$, of the interface between mixing species, e.g., the level set, $Z(\mathbf{x}, t)=Z_{0}=\langle Z\rangle_{\mathbf{x}}$. For a free shear layer, for example, $\langle Z\rangle_{\mathbf{x}}$ is set by the (volumetric) entrainment ratio, the ratio of high-speed to low-speed fluid entrained 
(Dimotakis 1986), $E_{V}$, i.e.,

$$
\langle Z\rangle_{\mathbf{x}}=Z_{E} \equiv \frac{E_{V}}{1+E_{V}} .
$$

In high-Re turbulence, where K41 scaling (Equation 3) is expected to apply, interfacial surface-to-volume ratio scales as, $\mathcal{S} \sim 1 / \lambda_{\min } \sim 1 / \lambda_{\mathrm{K}} \sim R e^{3 / 4} / \delta$. As a consequence, one might expect a strong dependence of mixing on $R e$. However, experiments at high- $R e$ indicate that the dependence of mixing on $R e$ ranges from weak to difficult to discern, and is easily masked by effects that may accompany changes in $R e$ in a particular flow, such as boundary or initial/inflow conditions (George 1989, Slessor et al. 1998). This observation can be reconciled with the scaling for $\mathcal{S}$, above, by noting that the inner K41 strain rate (reciprocal of $\mathrm{K} 41$ time) scales as $\sigma_{\mathrm{K}}=1 / t_{\mathrm{K}}=\sqrt{\varepsilon / \nu} \propto(\Delta U / \delta) R e^{1 / 2}$ and that inner-scale diffusionlayer thicknesses scale as $\lambda_{\mathcal{D}} \sim\left(\mathcal{D} / \sigma_{\mathrm{K}}\right)^{1 / 2}$ (cf. Batchelor 1959, and Equation 5 and related discussion).

If the volume fraction (thickness) occupied by mixed fluid is modeled as that occupied by diffusion layers of thickness $\lambda_{\mathcal{D}}$ astride the interfacial surface area $\mathcal{S}$ (per unit volume), as modeled above, we find to leading $\operatorname{order}(S c \approx 1$, i.e., $\mathcal{D} \approx v$ ) for a given flow (Dimotakis 1991),

$$
\frac{\delta_{\mathrm{m}}}{\delta} \propto \mathcal{S} \lambda_{\mathcal{D}}=\frac{R e^{3 / 4}}{\delta} \sqrt{\frac{\mathcal{D}}{\sigma_{\mathrm{K}}}} \approx R e^{3 / 4} \sqrt{\frac{v}{\delta \Delta U}} \operatorname{Re}^{-1 / 4} \neq \mathrm{fn}(\operatorname{Re}) .
$$

Weaker (e.g., logarithmic) dependencies on $R e$ are admissible in the argument. Although $\delta_{\mathrm{m}} / \delta$ may not be independent of $R e$ in high-Re flows, the argument indicates a dependence on $R e$ that is weaker than power-law, in accord with observation. We also recognize the result of a singular-perturbation problem: $\delta_{\mathrm{m}} / \delta$ is the product of $\mathcal{S} \rightarrow \infty$ and $\lambda_{\mathcal{D}} \rightarrow 0$, as $R e \rightarrow \infty$, in a manner that leaves the product (approximately) independent of $R e$.

If the dependence on $S c$ is to be captured for $S c>1$, the argument(s) must also be extended to the sub- $\lambda_{\mathrm{K}}$ regime. Preliminarily, at fixed $R e$, the limit of $S c \rightarrow \infty$ is not a singular perturbation problem (cf. Equation 8 in fixed flow and consider varying the fluid property $\mathcal{D} \rightarrow 0$ ) and we expect

$$
p(Z ; \mathbf{x} ; R e, S c \rightarrow \infty) \rightarrow[1-\langle Z(\mathbf{x})\rangle] \delta_{\mathrm{D}}(Z)+\langle Z(\mathbf{x})\rangle \delta_{\mathrm{D}}(1-Z)
$$

with $\langle Z(\mathbf{x})\rangle_{\mathbf{x}}=\int_{Z} Z p(Z ; \mathbf{x}) \mathrm{d} Z$ and $\delta_{\mathrm{D}}(Z)$ the Dirac delta function, and

$$
\frac{\delta_{\mathrm{m}}(\operatorname{Re}, S c \rightarrow \infty)}{\delta} \rightarrow 0 .
$$

The argument implicit in Equation 19 treats diffusion layers as disjoint. However, diffusion layers will merge at small scales, leading to the formation of nearly homogeneously mixed local regions. A proper tally must include the contribution to the mixed-fluid fraction of both separated and merged diffusion layers. 
Such a model was proposed by Broadwell \& Breidenthal (1982), who partitioned mixed fluid as residing in strained diffusion layers between unmixed entrained fluids and homogeneously mixed fluid. In a subsequent discussion, Broadwell \& Mungal (1991) specified that the diffusion layers should be modeled as subjected to the large-scale strain rate, $\sigma_{\delta} \sim U / \delta$, with a viscous thickness given by the Taylor scale (Equation $2 b$ ), and a homogeneously mixed fluid at the composition $Z=Z_{E}$ (Equation 18). The PDF from the Broadwell-BreidenthalMungal (BBM) model (integrated across the transverse coordinate, $y$ ) is then (for $Z \neq 0,1)$,

$$
p(Z) \mathrm{d} Z=\left[C_{\mathrm{H}} \delta_{\mathrm{D}}\left(Z-Z_{E}\right)+C_{\mathrm{F}} \frac{p_{\mathrm{F}}(Z)}{\sqrt{\operatorname{ReSc}}}\right] \mathrm{d} Z
$$

In this expression, $p_{\mathrm{F}}(Z)$ is the PDF of composition in strained laminar diffusion layers between $Z=0,1$ fluids (error-function $Z$-profile), and $C_{\mathrm{H}}$ and $C_{\mathrm{F}}$ are constants to be determined by fitting experimental data. Applying the BBM model $\mathrm{PDF}$ to a shear layer yields a product volume fraction,

$$
\frac{\delta_{\mathrm{P}}\left(Z_{\phi} ; R e, S c\right)}{\delta} \simeq C_{\mathrm{H}}+\frac{C_{\mathrm{F}}}{\sqrt{\operatorname{ReSc}}} F\left(Z_{\phi}\right), \quad F\left(Z_{\phi}\right) \equiv \frac{e^{-z_{\phi}^{2}}}{\sqrt{\pi} Z_{\phi}\left(1-Z_{\phi}\right)},
$$

with $\operatorname{erf}\left(z_{\phi}\right) \equiv(\phi-1) /(\phi+1)=2 Z_{\phi}-1$. The mixed-fluid fraction, $\delta_{\mathrm{m}} / \delta$, can then be computed using Equation 19. BBM assume a $R e$-independent contribution to $\delta_{\mathrm{m}} / \delta$ from homogeneously mixed fluid and predict a negligible contribution from diffusion layers in water. The model accommodates the decrease in $\delta_{\mathrm{m}} / \delta$ with increasing $S c$, observed in the comparison of gas-phase ( $S c \sim 1$, Mungal $\&$ Dimotakis 1984) versus liquid-phase $\left(S c \sim 10^{3}\right.$, Koochesfahani \& Dimotakis 1986) shear layers (factor of $\sim 2$ lower for liquid-phase mixing), at comparable flow conditions and $R e$ (cf., Dimotakis 1991). BBM model predictions are independent of viscosity $(R e)$ and depend on the Peclet number, $\mathrm{Pe} \equiv \delta U / \mathcal{D}=R e S c$, only, i.e., not on $R e$ and $S c$ separately. The BBM model has the limiting behavior, $\delta_{\mathrm{m}}(\operatorname{Re} \rightarrow \infty, S c) / \delta=\delta_{\mathrm{m}}(\operatorname{Re}, S c \rightarrow \infty) / \delta=C_{\mathrm{H}}=$ const., i.e., an algebraic $\operatorname{Re}$ dependence at fixed $S c$, and a $(S c \rightarrow \infty)$-limit, at fixed $R e$, not in accord with Equation 20b.

In an alternative proposal for mixing in high-Re shear layers (Dimotakis 1989), mixed fluid is modeled as in diffusion layers across the full turbulence $\lambda$ spectrum in a K41 strain-rate field: $\sigma\left(\lambda>\lambda_{\mathrm{c}}\right) \approx \sigma_{\mathrm{c}}\left(\lambda_{\mathrm{c}} / \lambda\right)^{2 / 3}, \sigma\left(\lambda<\lambda_{\mathrm{c}}\right)=\sigma_{\mathrm{c}}$, with $\lambda_{\mathrm{c}}=\beta^{3 / 2} \lambda_{\mathrm{K}}, \sigma_{\mathrm{c}}=v /\left(\beta \lambda_{\mathrm{K}}^{2}\right)$, and $\beta \simeq 3$. Diffusion layers are treated as spaced by $\lambda$ along space curves locally perpendicular to $Z_{E}$-isosurfaces, and merging at small $\lambda$ as part of a continuous process. The model assumes a statistical-weight contribution for each $\lambda$, given by $w\left(\lambda>\lambda_{\mathrm{c}}\right) \mathrm{d} \lambda \approx \mathrm{d} \lambda / \lambda$, consistent with $\mathrm{K} 41$ (no characteristic length in the inertial range), and $w\left(\lambda<\lambda_{\mathrm{c}}\right) \mathrm{d} \lambda \approx \mathrm{d} \lambda / \lambda_{\mathrm{c}}$; incorporates the K62 proposal for fluctuations in $\varepsilon$ (Kolmogorov 1962); and treats the shear-layer entrainment ratio, $E$, as log-normally distributed (Bernal 1988). The Dimotakis (1989) model computes the product thickness for a chemical reaction 
in the fast-kinetic regime, yielding,

$$
\frac{\delta_{\mathrm{P}}\left(Z_{\phi} ; R e, S c\right)}{\delta} \simeq \frac{B\left(Z_{\phi} ; S c\right)}{1+\ln \left(\beta S c^{q} / C_{\mathrm{B}}\right)+\frac{3}{4}\left(1-\frac{\mu}{8}\right) \ln \left(\operatorname{Re} / \operatorname{Re}_{\mathrm{cr}}\right)},
$$

where $C_{\mathrm{B}}=1.6$ (cf., Equation 4, and equation 2.38 and discussion in Dimotakis 1989); for $S c \gtrsim 1, q=1 / 2 ; B\left(Z_{\phi} ; S c\right)$ depends on whether diffusion-layer merging is expected at scales larger/smaller than $\lambda_{c}$, as estimated by the model, and weakly on $Z_{\phi}$, for small $Z_{\phi}(\epsilon$ in Equation 15$) ; \mu \simeq 0.20-0.45$ is the dissipation-rate fluctuation coefficient (Kolmogorov 1962; Monin \& Yaglom 1975, section 25; Oboukhov 1962), with values $\mu \approx 0.25$ (Van Atta \& Antonia 1980) and $\mu \approx$ 0.30 (Ashurst et al. 1987), the latter value assumed in Dimotakis (1989); and $R e_{\mathrm{cr}} \simeq 26$ (critical $R e$ for viscous free-shear layer transition). The mixed-fluid fraction, $\delta_{\mathrm{m}} / \delta$, is then estimated with Equation 17. Other than the choices outlined above, no adjustable parameters are employed. The model captures the difference in $\delta_{\mathrm{m}} / \delta$ between gas- and liquid-phase mixing (Dimotakis 1989, figure 26) and the predicted weak dependence on $R e$ is in accord with experiment, at least in the $R e$-range where data are available, as illustrated below. It also predicts that $\delta_{\mathrm{m}}(\operatorname{Re} \rightarrow \infty) / \delta \rightarrow 0$, albeit slowly (see Dimotakis 1989 and 1991 for further discussion).

Figure 3 depicts gas-phase $\delta_{\mathrm{P}} / \delta$ data for $4 \times 10^{4} \lesssim R e \lesssim 7 \times 10^{5}$, measured using the hydrogen-fluorine chemical reaction at $\phi=1 / 8\left(Z_{\phi} \simeq 0.11\right)$, in the

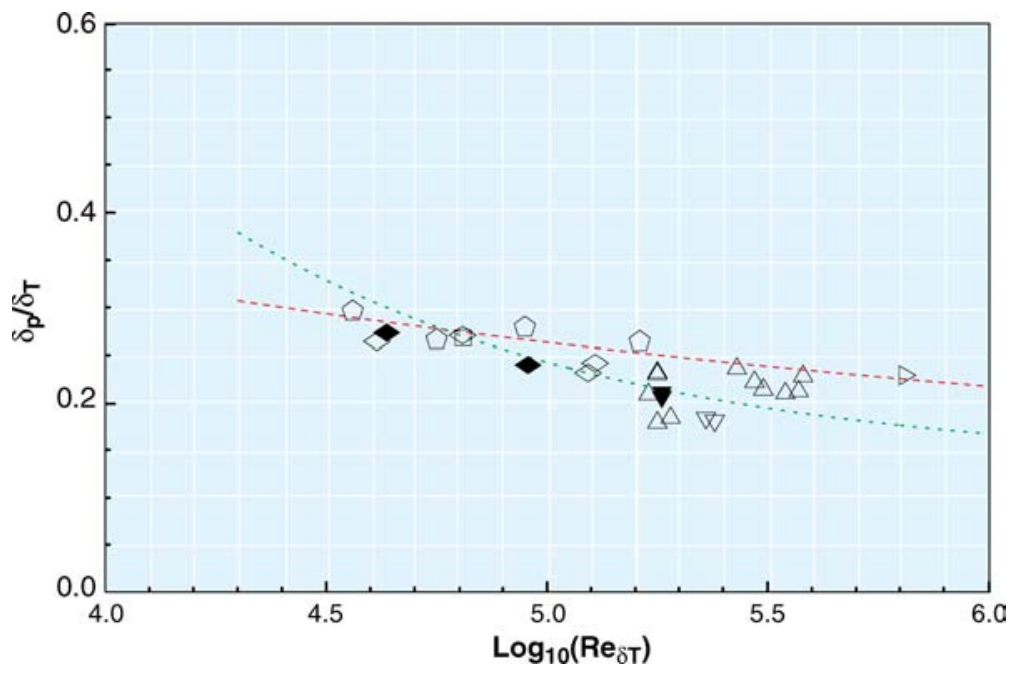

Figure 3 Chemical-product thickness versus $\operatorname{Re}$ at $\phi=1 / 8$. Square: Mungal $\&$ Dimotakis (1984), diamonds: Mungal et al. (1985), pentagons: Frieler (1992), uppointing triangles: Bond (1999), down-pointing triangle: Slessor (1998). Model predictions: Dotted (blue-shaded) line BBM, dashed (pink-shaded) line Dimotakis (1989). 
fast-kinetic regime, at low heat release (Bond 1999). The data indicate a decreasing chemical-product (mixed-fluid) mole fraction with increasing $R e$, at least in this $R e$ regime. Also plotted in Figure 3 are the predictions of the BBM model (dotted blue-shaded line), fitting the point at $R e \simeq 6.3 \times 10^{4}$ (Mungal \& Dimotakis 1984) and the point from the liquid-phase experiment by Koochesfahani \& Dimotakis (1986) at a comparable $R e$ to determine the $C_{\mathrm{H}}$ and $C_{\mathrm{F}}$ constants, as recommended by the authors (Equation 21), as well as the prediction of the Dimotakis (1989) model (dashed pink-shaded line). This flow exhibits a strong dependence on inflow conditions (boundary-layer state), in the range $5.2 \leq \log _{10}(R e) \leq 5.5$ (Slessor et al. 1998), rendering extrapolation to higher-Re on the basis of these data of questionable value and appreciate that higher-Re experimental data may not be in the offing. Considering the very large $R e$ range encountered in engineering and nature, this issue is important but may require resolution by other means.

Experimental evidence indicates that the (slight) decrease in mixing with increasing $R e$ may be peculiar to shear layers and not observed in post-mixingtransition turbulent jets, for example (Dimotakis 2000). Experiments (e.g., compilation in Dahm \& Dimotakis 1987) also suggest that mixing measures are less sensitive to $S c$ as well, indicating a rather different role for molecular transport coefficients in turbulent jet mixing. An example is "flame length", i.e., the number of nozzle diameters required to entrain and mix ambient fluid such that (all parts of) the jet-nozzle fluid are mixed to below a certain composition. Although the expected dependence of scalar dispersion and fluctuations on $S c$ are welldocumented (e.g., Taylor 1953, 1954; Batchelor 1959; Saffman 1960; Dowling \& Dimotakis 1990; Miller \& Dimotakis 1996; Papavasiliou \& Hanratty 1997; Warhaft 2000; Papavasiliou 2002; Villermaux 2002), differences in the sensitivity of mixing to molecular-transport coefficients at high- $R e$, between turbulent shear layers and jets, for example, suggest that universal theories of turbulent mixing will not likely prove successful. This is despite the fact that mixing is a small-scale phenomenon and hopes of universality for small-scale behavior and statistics often dot theoretical discussions.

\section{LEVEL 2: MIXING COUPLED TO DYNAMICS}

In Level-2 mixing, flow dynamics are altered by mixing processes. Examples arise in stably stratified and unstable inhomogeneous (variable-density) flows subjected to imposed pressure gradients, or external force/acceleration fields. An important characteristic that distinguishes such flows from Level-1 mixing is the generation of baroclinic vorticity that derives from misalignments between pressure and density gradients, or, equivalently, temperature and entropy gradients in the flow. Vorticity generated by this mechanism will drive internal Kelvin-Helmholtz layers and instability that can/will increase scalar- or density-isosurface generation and, by extension, mixing. Mixing is coupled to dynamics by homogenizing 
density variations (decreasing gradients), thereby altering local baroclinic vorticity generation.

Rayleigh-Taylor Instability (RTI) flows provide a good example of the coupling of mixing with the flow dynamics. These flows occur whenever a flow with a density gradient is subjected to acceleration (with a component) opposite this gradient (Rayleigh 1883; Taylor 1950; Chandrasekhar 1955, 1961; Sharp 1984). Emptying of an inverted glass of water is a consequence of RTI, as readily demonstrated by inverting a water-filled glass covered by a thin sheet of paper (suffices to impede interfacial instability growth; one atmosphere pressure supports $\approx 10 \mathrm{~m}$ of water). However, experiments are difficult and much of our knowledge on RTI mixing derives from computer simulations (DNS) at necessarily moderate $R e$ 's, at least at this writing (e.g., Cook \& Dimotakis 2001, 2002; Cook \& Zhou 2002).

A dynamic role that mixing plays in RTI illustrates a mechanism not uncommon in unstably stratified flows and can be appreciated by a useful model of RTI mixing-zone growth. RTI growth is driven by the conversion to kinetic energy of the potential energy in the originally inverted stratification (Fermi \& von Neumann 1955, Sharp 1984). Semi-quantitatively, it can be understood in terms of rising/falling buoyant bubbles/"spikes" driven by the density difference, $\Delta \rho$, across their fronts, in the presence of an acceleration field of magnitude $g$. The RTI mixing-zone extent is then the separation between advancing bubble and spike fronts, i.e., $\dot{h}=\dot{h}_{\mathrm{b}}+\dot{h}_{\mathrm{s}}=u_{\mathrm{b}}+u_{\mathrm{s}}$. Batchelor (1967) relaxed some of the restrictions in the original discussion by Davies \& Taylor (1950) deriving their terminal speed of advance,

$$
u_{\mathrm{b}, \mathrm{s}}=C_{\mathrm{bs}}\left(\frac{\Delta \rho}{\rho_{\mathrm{b}, \mathrm{s}}} g R_{\mathrm{b}, \mathrm{s}}\right)^{1 / 2},
$$

where $C_{\mathrm{bs}}$ is a geometry-dependent constant $(=2 / 3$ for locally spherical bubble/spike fronts), $\rho_{\mathrm{b}, \mathrm{s}}$ is the unmixed-fluid density ahead of the advancing bubble/spike, and $R_{\mathrm{b}, \mathrm{s}}$ is the radius of curvature at the bubble/spike stagnation point. At least at low to moderate $R e$ 's, where leading bubbles and spikes retain their structure as they advance, this model recovers classical RTI growth descriptions, i.e.,

$$
h_{\mathrm{b}, \mathrm{s}}(t) \simeq \alpha_{\mathrm{b}, \mathrm{s}} \mathcal{A} g\left(t-t_{0}\right)^{2},
$$

with $\mathcal{A} \equiv\left(\rho_{1}-\rho_{2}\right) /\left(\rho_{1}+\rho_{2}\right)$ the Atwood number and the $\alpha_{\mathrm{b}, \mathrm{s}}$ accepted as densityratio-dependent constants. (Dimonte \& Schneider 2000), if $\left\langle R_{\mathrm{b}, \mathrm{s}}\right\rangle \propto h_{\mathrm{b}, \mathrm{s}}$, as follows from turbulence similarity in the absence of imposed outer length scales. Unmixed fluid is entrained via the bubble/spike rear and convected to the interior side of the interface in the vicinity of the stagnation point. Mixing decreases $\Delta \rho$ across the front, decreasing, in turn, $u_{\mathrm{b}, \mathrm{s}}$ and, in this model, RTI mixing-zone growth. Mixing also alters the anisotropic (vertical) buoyancy forces that act throughout the spectrum of scales through its influence on the internal 
inhomogeneous fluid parcel composition. Such forces can break the tendency toward small-scale isotropy in a manner likely to depend on the quality of mixing, complicating turbulence modeling that would otherwise benefit from isotropy.

Dynamics and mixing in stably stratified flows present special modeling challenges and introduce a host of additional space/time scales (Riley \& Lelong 2000). In such flows, isopycnal (uniform-density) surface elevation perturbations (can) generate (internal) waves that can have nonlocal consequences. Isopycnal dispersion and mixing, i.e., within iso-density surfaces, can be approximated with 2D turbulence constructs (e.g., Pasquero et al. 2001). Diapycnal dispersion and mixing, i.e., across isopycnal surfaces, however, presents a difficult modeling challenge and "... has remained enigmatic" (Peltier \& Caulfied 2003). It requires a minimum value for the gradient Richardson number that is based on the buoyancy (Brunt-Väisälä) frequency, $N(z)$, and vertical shear, i.e.,

$$
R i_{\mathrm{g}} \equiv \frac{N^{2}(z)}{\partial_{z}\left|\mathbf{u}_{\perp}\right|^{2}}=\frac{-g \partial_{z} \bar{\rho}(z) / \rho}{\left(\partial_{z} u\right)^{2}+\left(\partial_{z} v\right)^{2}}
$$

where $\mathbf{u}_{\perp}$ is the horizontal velocity (components perpendicular to the density gradient), $g$ the gravitational/imposed acceleration, and $\bar{\rho}(z)$ the mean stratified density profile. For $R e_{\mathrm{g}}>1 / 4$, stratified parallel flows are stable to small disturbances (Howard 1961, Miles 1961). They undergo a (stably stratified) mixing transition for $R e_{\mathrm{g}}<1 / 4-1$ (Peltier \& Caulfied 2003), when an adequate (horizontal) kinetic energy gradient (vertical shear) renders vertical mixing energetically feasible. Important examples where mixing and dispersion play a key role arise in stably stratified flows, in both ocean (e.g., Tziperman 1986) and atmosphere flows (e.g., Smith et al. 2002, Lapeyre \& Held 2003, Schneider 2004, and references therein).

An important special example is Langmuir circulation (LC) that forms near surface layers of oceans, lakes, and ponds (Langmuir 1938) with winds of moderate strength. Especially following extensive observations in the Pacific (Smith 1992, 1998), LC is credited for significant transport of mass, momentum, and heat through the upper-ocean mixed layer that is key in air-sea exchange and the weather. LC forms cellular arrays of near-parallel counter-rotating vortical cells, aligned with the wind direction, that concentrate flotsam, seaweed, and air bubbles into clearly visible streaks. LC-cell spacings range from millimeters to hundreds of meters, or, possibly, kilometers (Thorpe 2004). LC cells are thought to arise through an inviscid instabilty driven by a nonlinear interaction of gravity waves, wind-induced shear, and Stokes drift (Phillips 2001a,b, and 2002), with LC spacing set inviscidly (Phillips 2004), at least in the absence of wave breaking and strong turbulence. As with mixing in other stably stratified flows, LC formation requires a minimum driving force, e.g., surface wind speed (Veron \& Melville 2001). LC onset, transport, and mixing represent significant components of the air-sea interfacial exchange, and near-surface ocean transport and mixing, and must be captured by global change models. 


\section{LEVEL 3: MIXING THAT ALTERS THE FLUID}

In Level-3 mixing, the flow is altered by attendant changes in the fluid. Combustion is probably the most familiar example, imposing additional time/space scales and phenomenology that contribute considerable complexity to the dynamics and substantial space-time stiffness to numerical-simulation approaches. In the context of turbulent mixing, the focus here is on gas-phase combustion and sets aside important issues such as particles (soot, droplets) and radiation, to help circumscribe the discussion.

The main element introduced by combustion and related phenomena is (complex) kinetics. These enter through highly nonlinear reaction rates in the scalar transport equations (represented by $\varpi_{\alpha}$ in Equation 7). The typical Arrhenius temperature and species-concentration dependence leads to a tightly coupled set of transport equations (e.g., Williams 1985, Peters 2000), one for each species, $\alpha$. Hydrocarbon combustion - an important special case-involves tens to hundreds of species and hundreds (e.g., for methane-air combustion) to thousands of elementary reactions (e.g., for octane and higher-C hydrocarbons), with chemical species that are reactants, products, or radicals. Of these, radicals (e.g., $\mathrm{H}, \mathrm{OH}$, $\mathrm{CH}, \ldots$ ) are special, if not key, to combustion and by being created and consumed more or less in place, do not participate in mixing, in the main sense of this paper. However, they can be highly reactive and must be tracked with some fidelity. They can also be responsible for significant differential-diffusion effects, e.g., hydrogen atoms $(\mathrm{H})$. Radicals exist around active reaction zones that, for the typical large activation temperatures in hydrocarbon fuels, are quite thin (adding considerable spatial stiffness to any simulation) but play a vital role by linking reactants to products in complex hydrocarbon reaction networks.

Turbulent mixing plays a crucial role in nonpremixed combustion, with initially segregated reactants (Vervisch \& Poinsot 1998). Turbulent mixing is also important in premixed combustion where imperfect mixing of species and of other inhomogeneities alters the propagation speed of combustion waves, such as flames, deflagrations, or even detonations. These phenomena, referred to as the turbulencechemistry interaction, form a tightly coupled relationship. Mixing brings reactants together, a precursor to chemical conversion, leading to product formation and energy release (enthalpy conversion). Such changes alter the temperature and density, mean molar mass, specific heat capacity and ratio, and mixture transport properties. Changes in density are immediately felt, influencing momentum transport and pressure relaxation, entrainment, and mixture-fraction PDFs (e.g., Batt 1977, Kennedy \& Kent 1978, Wallace 1981, Hermanson \& Dimotakis 1989, Miller et al. 1998). Realistic variable-density values were only recently investigated using DNS (Pantano et al. 2003). The typical increase of molecular transport values with temperature also decreases local $R e$ 's, modifying mixing. These changes, primarily the result of variable density and temperature, impact the large scales of the turbulence. These control entrainment, are strongly coupled to buoyancy effects, 
and determine, in turn, the small-scale environment where mixing and combustion ultimately occur.

Nonlinear reaction rates have a gradient-steepening effect on reactants, altering diffusive fluxes and mixing. At certain flow locations, where temperatures are sufficiently high to support rapid chemical conversion, reactants and radicals are consumed, steepening gradients and altering mass transport of the major species beyond values that would occur in cold flows. Scant quantitative information is available on such phenomena. Some theoretical and experimental work indicates changes in scalar spectra of one-step chemical reactions, mostly for constantdensity flows. For first-order reactions, results from this work (e.g., Corrsin 1961, 1964; Toor 1962; Pao 1964; O’Brien 1968; Hill 1976; Lundgren 1985) indicate changes at high wavenumbers in scalar spectra that are not observed in passive scalars. Coherence spectra measurements for second-order reactive scalars, with negligible heat release, indicate high coherence between reactants for low wavenumbers, ranging to incoherence and random phase at higher wavenumbers (e.g., Bilger et al. 1991, Kosaly 1993, de Bruyn Kops et al. 2001). However, little is known about spectra of reactive scalars for realistic chemistry and levels of heat release.

The BBM and Dimotakis (1989) models (section 5) highlight the importance of strain rate imposed on diffusion layers and mixing. The outer-scale strain rate for the gas-phase shear layer in the example above is given by $\sigma_{\delta}=\Delta U / \delta=$ $1.7 \times 10^{3} \mathrm{~s}^{-1}$, while the inner-scale $\mathrm{K} 41$ strain rate, $\sigma_{\mathrm{K}} \approx \sigma_{\delta} R e^{1 / 2} \approx 5.3 \times 10^{5} \mathrm{~s}^{-1}$. These numbers present a challenge for modeling and experiments on mixing accompanied by chemical reactions and combustion. The gas-phase mixing measurements summarized in Figure 3 relied on the fast hydrogen (premixed with nitric oxide) and fluorine chemical hypergolic system. Gas-phase combustion, however, typically involves slower-kinetic reactants with activation energies substantially higher than $k_{\mathrm{B}} T$, require ignition and flameholding, and are subject to extinction (local quenching).

For most combustion, the competition between finite chemical rates and mixing rates (local strain rates) has important consequences and merits special mention. The relative importance of these two time scales is parameterized by $D a$ (Equation 6). When this is small, at high strain rates or slow chemistry, combustion of typical (nonhypergolic) reactants is usually locally quenched. This results in local flame extinction, forming flame edges, triple-edge flames (Dold 1988), and holes (cf., review by Buckmaster 2002), possibly followed by local spontaneous reignition. Figure 4 (Pantano 2004) depicts a three-dimensional (3D) nonpremixed methane-air flame, as marked by the local $\mathrm{H}$-atom concentration, derived from a numerical simulation using the Peters (1985) four-step mechanism. High $\mathrm{H}$-atom concentration (red) marks intense burning associated with closing flame holes, for example. Such flame events are tightly coupled to the flow and poorly understood mainly because of the complex interplay between chemistry, local flame topology and dilatation, and mixing. 


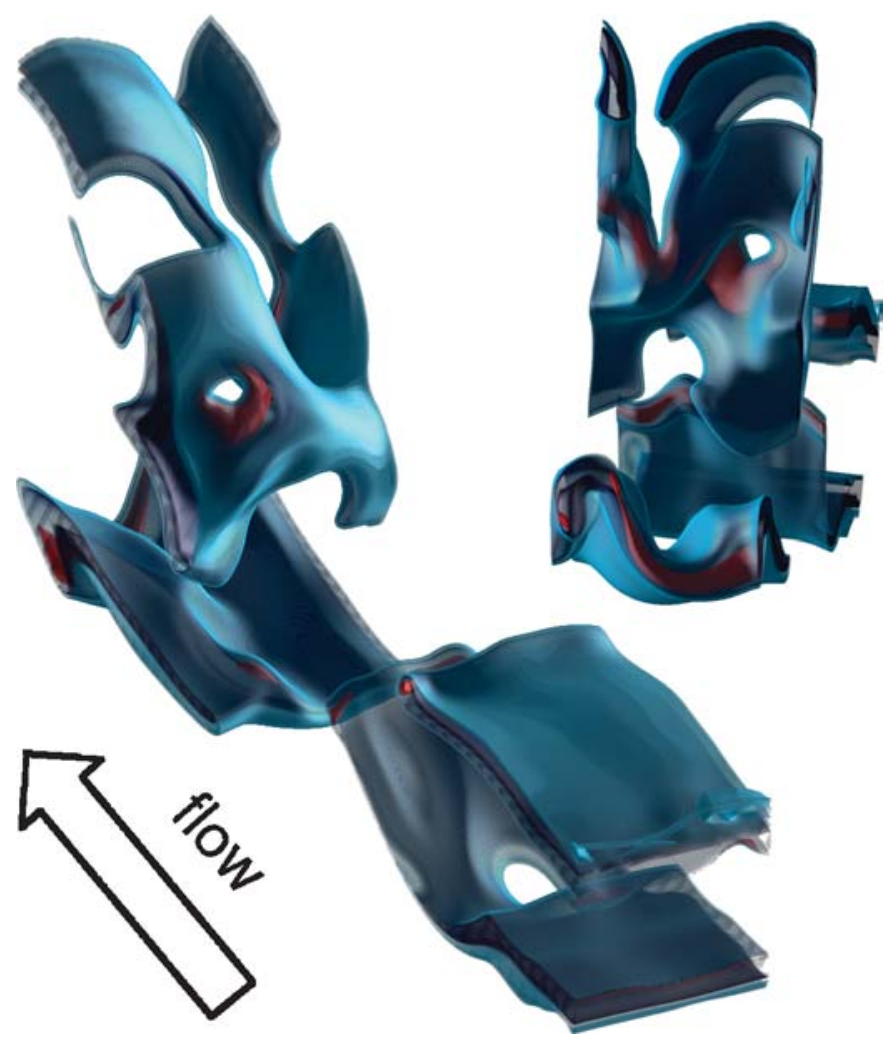

Figure 4 Flame edges and holes in three-dimensional combustion of methane with entrained air, issuing from a piloted slot burner (Pantano 2004). Color denotes H-atom concentration.

\section{THEORY, MODELING, AND SIMULATION}

From a theoretical, modeling, and simulation perspective, if a universal/quasiuniversal theoretical approach does not appear promising (section 5) and we must also accept DNS as an infeasible option for high-Re flows (section 2), as widely acknowledged, other approaches must be sought. A more promising approach may be to address the multiscale and numerical-simulation stiffness problems of turbulent mixing. This requires models of the response and coupling of small-scale dynamics and mixing to those at larger scales. Such models can then be used in simulations that, in the interest of tractability, are purposely too coarse to resolve the full spectrum of space-time scales. Many such efforts are in progress at this time whose assessment is beyond the scope of the present review. The approach that has, perhaps, shown the most promise to date relies on Large Eddy Simulation (LES), augmented by Sub-Grid Scale (SGS) modeling (Lesieur \& Métais 1996). 
Briefly, we consider the Favre-filter of any field $f(\mathbf{x}, t)$, i.e., $\tilde{f} \equiv \overline{\rho f} / \bar{\rho}$, where $\bar{f} \equiv \int G\left(\mathbf{x}-\mathbf{x}^{\prime}\right) f\left(\mathbf{x}^{\prime}\right) \mathrm{d} \mathbf{x}^{\prime}$ denotes the underresolved component of $f(\mathbf{x}, t)$. Applying the Favre filter to incompressible homogeneous flow, we have,

$$
\partial_{i} \tilde{u}_{i}=0, \quad \partial_{t} \tilde{u}_{i}+\partial_{j}\left(\tilde{u}_{i} \tilde{u}_{j}\right)=f_{i}-\frac{1}{\rho} \partial_{i} \tilde{p}+v \nabla^{2} \tilde{u}_{i}-\partial_{j} \tau_{i j},
$$

where $f_{i}$ is the sum of the (resolved) body forces and $\tau_{i j} \equiv \widetilde{u_{i} u_{j}}-\tilde{u}_{i} \tilde{u}_{j}$ is the SGS stress tensor (Leonard 1974, 1997). A similar decomposition can be applied to the transport equation for a passive scalar. Considering mixing in a two-fluid system with a uniform Fickian diffusivity, $\mathcal{D}=$ const., for example, we may write for the mole-fraction conservation equation,

$$
\partial_{t} \tilde{X}+\partial_{i}\left(\tilde{u}_{i} \tilde{X}\right)=\mathcal{D} \nabla^{2} \tilde{X}-\partial_{i} \zeta_{i},
$$

with $\zeta_{i}$ the SGS flux vector of $\tilde{X}$ (Pullin 2000). Closure of these equations requires a model for $\tau_{i j}$ and $\zeta_{i}$ that must capture the effects of the unresolved dynamics, stresses, and fluxes on the resolved flow, and vice versa, in terms of resolved fields. This is a multiscale modeling challenge, whose details will depend on the resolved fraction of the scale spectrum in the simulation, which spans all the way from the outer scale, $\delta$, to some scale larger than the inner viscous scales, $\lambda_{\nu}$ or $\lambda_{K}$, or diffusion scales, $\lambda_{\mathcal{D}}$ or $\lambda_{\mathrm{B}}$, and, in particular, on where the spatial-averaging cut-off scale, $\Delta$, of the effective averaging kernel, $G\left(\mathbf{x}-\mathbf{x}^{\prime}\right)$, lies within this spectrum. A requirement for such a model is that it must revert to the equations for fully resolved dynamics, whereever and whenever $\Delta$ is sufficiently small.

Such modeling can rely on extrapolations of velocity/scalar behavior to the smaller, sub-grid scales. Extrapolations derived from fluid dynamics could be based on small-scale behavior derived from larger- and resolved-scale structurefunction information (e.g., Danaila et al. 2002; Lundgren 2002, 2003), as argued by Meneveau \& O'Neil (1994); or on ideas from fractal analysis (e.g., Sreenivasan 1991), or on more general irregular level-set geometric properties and similarity (e.g., Dimotakis \& Catrakis 1999), as in Scotti \& Meneveau (1999); general optimization models that attempt to minimize the error between modeled and actual SGS dynamics (Moser \& Zandonade 2003); scale-dependent dynamic models that observe the Germano et al. (1991) identity (also Germano 1992), with some success in several flows (e.g., Meneveau et al. 1996, Moin et al. 1991, and Porte-Agel et al. 2000); and many other schemes and proposals (cf., review by Lesieur \& Métais 1996) beyond the scope of this brief discussion.

One SGS model that specifically addresses mixing with promising results is described in Voelkl et al. (2000) and Pullin (2000) and is based on extensions to the stretched-vortex model (Misra \& Pullin 1997, Pullin \& Saffman 1994). It represents SGS dynamics assuming that unresolved flow and dynamics within each simulation cell can be represented by a suitably aligned Lundgren (1982) spiral vortex. SGS mixing is modeled assuming that these vortices wrap the SGS scalar field to produce the SGS scalar fluxes and fluctuations. Figure 5 plots the normalized variance computed by Pullin (2000) for a scalar with an imposed mean 


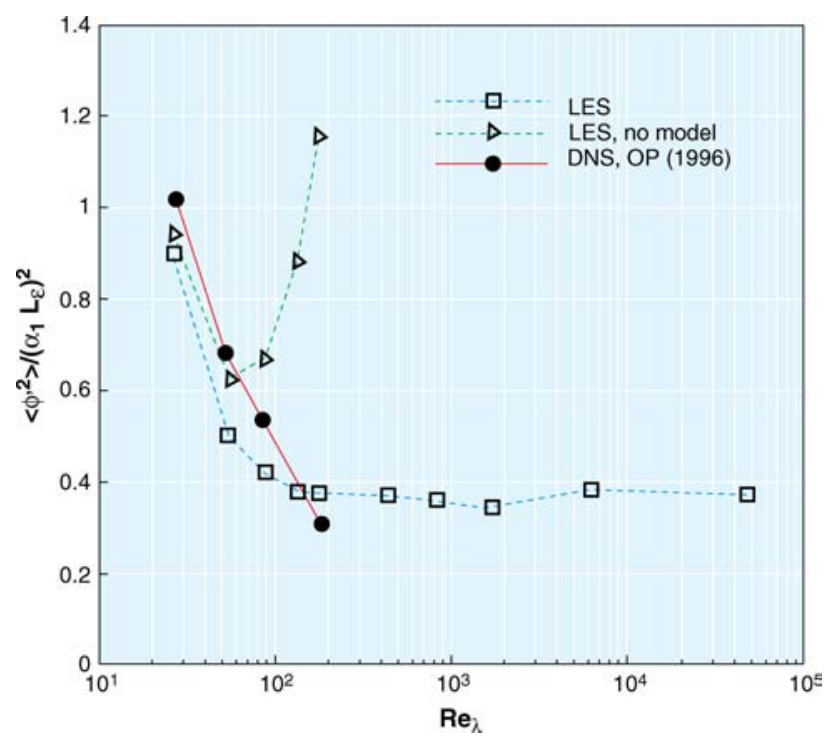

Figure 5 LES/SGS results (Pullin 2000, figure 3) of the normalized variance of a scalar with an imposed mean gradient, transported by isotropic turbulence. Comparison with DNS results of Overholt \& Pope (1996).

gradient for $S c=0.7$; an important problem addressed via DNS by Overholt \& Pope (1996). The results are in accord with the DNS results, as a function of $R e_{\mathrm{T}}$. The LES-SGS simulation also captures the mixing transition, at $R e_{\mathrm{T}} \approx 100$, even though there is nothing in the SGS modeling to induce this particular attribute. In particular, at low $R e_{\mathrm{T}}$, scalar fluctuations decrease with increasing $R e_{\mathrm{T}}$, i.e., better mixing, transitioning to a regime with a weak, if any, $R e_{\mathrm{T}}$ dependence, as expected from arguments in the discussion in section 4 . This model was extended to mixing in high-Sc flows, capturing the predicted (Batchelor 1959) $k^{-1}$ spectrum (Pullin \& Lundgren 2001).

\section{DISCUSSION AND CONCLUDING REMARKS}

Sustained passive scalar mixing requires no power other than that necessary to maintain turbulence against (the small) viscous dissipation (at high $R e$ ). Most work on mixing has focused on this regime and has not addressed mixing-related energetics. In particular, mixing in unstably stratified flows is a power beneficiary, with kinetic energy derived from available potential energy through (vertical) overturning, and is energetically favored. In contrast, diapycnal mixing in stably stratified flows must overcome the potential-energy costs of vertical overturning per unit time, as well as the attendant internal-wave generation, and requires power sources 
(substantially) larger than those required to overcome the (small) viscous-dissipation losses. In the atmosphere, mixing would primarily occur in the planetary boundary layer, jet stream (clear-air turbulence), and as a consequence of moistair/cloud dynamics, were it not for important energetic events such as storms and hurricanes. A similar picture emerges in ocean mixing, which could well be dominated by ocean-current wakes and stirring at rough bottom and shelf topography, and internal-wave breaking. In deep ocean flows, the power budget that sustains this process is an important issue in ocean and global dynamics and a research topic (Wunsch \& Ferrari 2004). Level-3 mixing, as in the case of combustion briefly discussed here, can tap energy sources far exceeding the kinetic energy of the flow that brings reactants together and can alter the dynamics in qualitative and quantitative ways. Under these circumstances, coupling between mixing and flow dynamics can be very strong, especially in confined flows, where dilatation can induce large pressure rise/gradients that are exploited for propulsion, explosions, etc.

Regarding future progress, the discussion here places the greatest hopes for understanding turbulence and mixing on experiments and LES-SGS modeling and computation, with much that is fruitful and important remaining to be done. Experimentally, the combination of continuously improving optical/laser diagnostics, digital-imaging, and data-acquisition techniques holds the promise of sufficiently resolved, high-quality, 3D data as a function of time, in moderate- to posttransitional-Re flows. Such experimental data can provide the means to probe the full dimensionality of turbulence and mixing, perhaps for the first time, permitting validations of turbulence and SGS models that are not constrained to comparisons with simple average profiles or low-order statistics, focusing on observations more closely related to mixing. Regarding theory, modeling, and simulations, one can easily argue that ever-faster computing, although welcome, will not by itself produce sufficient near-term progress. Considering LES-SGS, the discussion here also places hopes on models that respect physical SGS dynamics and correctly model the response to anisotropy, density inhomogeneneity, etc. Such models are needed to represent SGS dynamics in stratified and accelerated flows, and to incorporate such SGS contributions as baroclinic vorticity generation, extensions to the idiosyncrasies of near-wall turbulence, dilatation, as well as realistic scalar PDF modeling coupled with improved reduced-kinetics schemes for combustion. Such extensions will be necessary to address Level-2 and Level-3 mixing, as well as mixing in compressible turbulent flows.

\section{ACKNOWLEDGMENTS}

Support for this review by AFOSR Grants F49620-01-1-0006 and FA9550-04-10020, the DOE/Caltech ASCI/ASAP subcontract B341492, and the Caltech John K. Northrop Chair is gratefully acknowledged. I would also like to acknowledge both recent and previous discussions with and assistance by J. Adkins and W.R.C. Phillips on ocean mixing; D. Arnett, H. Bethe, and R. Lovelace on astrophysics and supernova explosions; J.E. Broadwell and M.G. Mungal on passive-scalar 
mixing; A.W. Cook, T.W. Mattner, D.I. Meiron, and P.L. Miller on RTI flow; H.J.S. Fernando, A. Mahalov, and J. Riley on mixing in stably stratified flows; H. Lam on scalar transport and diffusion; H.W. Liepmann on turbulence; C. Pantano on mixing and combustion dynamics; T.W. Mattner and D.I. Pullin on sub-grid scale modeling; and R.A. Shaw on particle and cloud dynamics; as well as assistance with the text by J.M. Bergthorson and D.I. Pullin.

\section{The Annual Review of Fluid Mechanics is online at http://fluid.annualreviews.org}

\section{LITERATURE CITED}

Adkins JF, McIntyre K, Schrag DP. 2002. The salinity, temperature, and $\delta^{18} \mathrm{O}$ of the glacial deep ocean. Science 298:1769-73

Ashurst WT, Kerstein AR, Kerr RM, Gibson CH. 1987. Alignment of vorticity and scalar gradient with strain rate in simulated NavierStokes turbulence. Phys. Fluids 30:234353

Batchelor GK. 1959. Small-scale variation of convected quantities like temperature in turbulent fluid. Part 1. General discussion and the case of small conductivity. J. Fluid Mech. 5:113-33

Batchelor GK. 1967. An Introduction to Fluid Dynamics. Cambridge, UK: Cambridge Univ. Press

Batt RG. 1977. Turbulent mixing of passive and chemically reacting species in a low-speed shear layer. J. Fluid Mech. 82:53-95

Bethe HA, Wilson JR. 1985. Revival of a stalled supernova shock by neutrino heating. Astrophys. J. 295:14-23

Bernal LP. 1988. The statistics of the organized vortical structure in turbulent mixing layers. Phys. Fluids 31:2533-43

Bilger RW, Saetran LR, Krishnamoorthy LV. 1991. Reaction in a scalar mixing layer. $J$. Fluid Mech. 233:211-42

Bond CL. 1999. Reynolds Number Effects on Mixing in the Turbulent Shear Layer. $\mathrm{PhD}$ thesis. Calif. Inst. Technol.

Broadwell JE, Breidenthal RE. 1982. A simple model of mixing and chemical reaction in a turbulent shear layer. J. Fluid Mech. 125:397-410

Broadwell JE, Mungal MG. 1991. Large-scale structures and molecular mixing. Phys. Fluids 3:1193-206

Buckmaster J. 2002. Edge-flames. Prog. Energ. Combust. Sci. 28:435-75

Burrows A. 2000. Supernova explosions in the universe. Nature 403:727-33

Chandrasekhar S. 1955. The character of the equilibrium of an incompressible heavy viscous fluid of variable density. Proc. Cambridge Philos. Soc. 51:162-78

Chandrasekhar S. 1961. Hydrodynamic and Hydromagnetic Stability. Oxford: Oxford Univ. Press. 1981. New York: Dover

Colgate SA, White RH. 1966. The hydrodynamic behavior of supernova explosions. Astrophys. J. 143:626-81

Cook AW, Dimotakis PE. 2001. Transition stages of Rayleigh-Taylor instability between miscible fluids. J. Fluid Mech. 443:6999

Cook AW, Dimotakis PE. 2002. Corrigendum-Transition stages of Rayleigh-Taylor instability between miscible fluids. J. Fluid Mech. 457:410-11

Cook AW, Zhou Y. 2002. Energy transfer in Rayleigh-Taylor instability. Phys. Rev. E 66:026312

Corrsin S. 1961. The reactant concentration spectrum in turbulent mixing with first-order reaction. J. Fluid Mech. 11:407-16

Corrsin S. 1964. Further consideration of Onsager's cascade model for turbulent spectra. Phys. Fluids 7:1156-59

Dahm WJA, Dimotakis PE. 1987. Measurements of entrainment and mixing in turbulent jets. AIAA J. 25:1216-23 
Danaila L, Anselmet F, Antonia RA. 2002. An overview of the effect of large-scale inhomogeneities on small-scale turbulence. Phys. Fluids 14:2475-84

Danaila L, Mydlarski L. 2001. Effect of gradient production on scalar fluctuations in decaying grid turbulence. Phys. Rev. E 64:016316

Davies RM, Taylor GI. 1950. The mechanics of large bubbles rising through extended liquids and through liquids in tubes. Proc. $R$. Soc. London Ser. A 200:375-90

de Bruyn Kops SM, Riley JJ, Kosaly G. 2001. Direct numerical simulation of reacting scalar mixing layers. Phys. Fluids 13:1450-65

Dimonte G, Schneider M. 2000. Density ratio dependence of Rayleigh-Taylor mixing for sustained and impulsive acceleration histories. Phys. Fluids 12:304-21

Dimotakis PE. 1986. Two-dimensional shearlayer entrainment. AIAA J. 24:1791-96

Dimotakis PE. 1989. Turbulent shear layer mixing with fast chemical reactions. In Turbulent Reactive Flows. Lect. Notes Eng. 40:417-85. New York: Springer-Verlag

Dimotakis PE. 1991. Turbulent free shear layer mixing and combustion. In High Speed Flight Propulsion Systems. Prog. Astronaut. Aeronaut. 137:265-340

Dimotakis PE. 2000. The mixing transition in turbulent flow. J. Fluid Mech. 409:69-98

Dimotakis PE. 2001. Recent advances in turbulent mixing. In Mechanics for a New Millennium, ed. H Aref, JW Phillips, pp. 327-44. Dordrecht: Kluwer

Dimotakis PE, Catrakis HJ. 1999. Turbulence, fractals, and mixing. In Mixing: Chaos and Turbulence, ed. H Chate, E Willermaux, JM Chomaz, pp. 59-143. New York: Kluwer

Dimotakis PE, Miake-Lye RC, Papantoniou DA. 1983. Structure and dynamics of round turbulent jets. Phys. Fluids 26:318592

Dold JW. 1988. Flame propagation in a nonuniform mixture: the structure of anchored triple flames. Prog. Astro. Aero. 113:240-48

Dowling DR, Dimotakis PE. 1990. Similarity of the concentration field of gas-phase turbulent jets. J. Fluid Mech. 218:109-41

Eckart C. 1948. An analysis of the stirring and mixing processes in incompressible fluids. $J$. Mar. Res. 7:265-75

Falkovich G, Fouxon A, Stepanov MG. 2002. Acceleration of rain initiation by cloud turbulence. Nature 419:151-54

Fermi E, von Neumann J. 1955. Taylor instability of incompressible liquids. Los Alamos Sci. Lab. Rep. AECU-2979

Frieler CE. 1992. Mixing and reaction in the subsonic 2-D turbulent shear layer. $\mathrm{PhD}$ thesis. Calif. Inst. Technol.

George WK. 1989. The self-preservation of turbulent flows and its relation to initial conditions and coherent structures. In Advances in Turbulence, ed. WK George, R Arndt, pp. 39-74. New York: Hemisphere

Germano M. 1992. Turbulence, the filtering approach. J. Fluid Mech. 238:325-36

Germano M, Piomelli U, Moin P, Cabot W. 1991. A dynamic subgrid-scale eddy viscosity model. Phys. Fluids 3:1760-65

Glezer A. 1988. The formation of vortex rings. Phys. Fluids 31:3532-42

Hermanson JC, Dimotakis PE. 1989. Effects of heat release in a turbulent, reacting shear layer. J. Fluid Mech. 199:333-75

Hill JC. 1976. Homogeneous turbulent mixing with chemical reaction. Annu. Rev. Fluid Mech. 8:135-61

Hirschfelder JO, Curtiss CF, Bird RB. 1954. Molecular Theory of Gases and Liquids. New York: Wiley

Howard LN. 1962. Note on a paper of John W. Miles. J. Fluid Mech. 10:509-12

Janka H-Th. 2002. The secrets behind supernovae. Science 297:1183-84

Jimenez J, Wray AA. 1998. On the characteristics of vortex filaments in isotropic turbulence. J. Fluid Mech. 373:255-85

Jimenez J, Wray AA, Saffman PG, Rogallo RS. 1993. The structure of intense vorticity in homogeneous isotropic turbulence. J. Fluid Mech. 255:65-90

Kennedy IM, Kent JH. 1978. Measurements of a conserved scalar in turbulent jet 
diffusion flames. Proc. Combust. Inst. 17: 279-87

Kolmogorov AN. 1941. Local structure of turbulence in an incompressible viscous fluid at very high reynolds numbers. Dokl. Akad. Nauk SSSR 30:301-5

Kolmogorov AN. 1962. A refinement of previous hypotheses concerning the local structure of turbulence in a viscous incompressible fluid at high Reynolds number. J. Fluid Mech. 13:82-85

Koochesfahani MM, Dimotakis PE. 1986. Mixing and chemical reactions in a turbulent liquid mixing layer. J. Fluid Mech. 170:83112

Kosaly G. 1993. Frequency spectra of reactant fluctuations in turbulent flows. J. Fluid Mech. 246:489-502

Landau LD, Lifshitz EM. 1997. Course of Theoretical Physics, Vol. 6. Fluid Mechanics London: Pergammon. 2nd ed.

Langmuir I. 1938. Surface motion of water induced by wind. Science $87: 119-23$

Lapeyre G, Held IM. 2003. Diffusivity, kinetic energy dissipation, and closure theories for the poleward eddy heat flux. J. Atmos. Sci. 60:2907-16

Leonard A. 1974. Energy cascade in largeeddy simulations of turbulent fluid flows. Adv. Geophys. 18:237-49

Leonard A. 1997. Large-eddy simulation of chaotic convection and beyond. AIAA Paper 97-0204

Lesieur M, Métais O. 1996. New trends in largeeddy simulations of turbulence. Annu. Rev. Fluid Mech. 28:45-82

Lundgren TS. 1982. Strained spiral vortex model for turbulent fine structure. Phys. Fluids 25:2193-203

Lundgren TS. 1985. The concentration spectrum of the product of a fast bimolecular reaction. Chem. Eng. Sci. 40:1641-52

Lundgren TS. 2002. Kolmogorov two-thirds law by matched asymptotic expansion. Phys. Fluids 14:638-42

Lundgren TS. 2003. Kolmogorov turbulence by matched asymptotic expansions. Phys. Fluids 15:1074-81
Meneveau C, Lund T, Cabot W. 1996. A Lagrangian dynamic subgrid-scale model of turbulence. J. Fluid Mech. 319:353-85

Meneveau C, O'Neil J. 1994. Scaling laws of the dissipation rate of turbulent subgrid-scale kinetic energy. Phys. Rev. E 49:2866-74

Meshkov EE. 1969. Instability of the interface of two gases accelerated by a shock wave. Sov. Fluid Dyn. 4:101-4

Miles JW. 1961. On the stability of heterogeneous shear flows. J. Fluid Mech. 10:496508

Miller MF, Bowman CT, Mungal MG. 1998. An experimental investigation of the effects of compressibility on a turbulent reacting mixing layer. J. Fluid Mech. 356:25-64

Miller PL, Dimotakis PE. 1996. Measurements of scalar power spectra in high Schmidt number turbulent jets. J. Fluid Mech. 308:129-46

Misra A, Pullin DI. 1997. A vortex-based subgrid stress model for large-eddy simulation. Phys. Fluids 9:2443-54

Moin P, Squires K, Cabot W, Lee S. 1991. A dynamic subgrid-scale model for compressible turbulence and scalar transport. Phys. Fluids 3:2746-57

Monin AS, Yaglom AM. 1975. Statistical Fluid Mechanics: Mechanics of Turbulence, ed. $\mathrm{J}$ Lumley, Vol. II. Cambridge, MA: MIT Press

Moser RD, Zandonade P. 2003. Development of high Reynolds number optimal LES models. In Reynolds Number Scaling in Turbulent Flow, ed. AJ Smits, pp. 169-74. Dordrecht: Kluwer

Mungal MG, Dimotakis PE. 1984. Mixing and combustion with low heat release in a turbulent mixing layer. J. Fluid Mech. 148:349-82

Mungal MG, Hermanson JC, Dimotakis PE. 1985. Reynolds number effects on mixing and combustion in a reacting shear layer. AIAA J. 23:1418-23

Oboukhov AM. 1962. Some specific features of atmospheric turbulence. J. Fluid Mech. 13:77-81

O'Brien EE. 1968. Lagrangian history direct interaction equations for isotropic turbulent mixing under a second-order chemical reaction. Phys. Fluids 11:2328-35 
Overholt MR, Pope SB. 1996. Direct numerical simulation of a passive scalar with imposed mean gradient in isotropic turbulence. Phys. Fluids 8:3128-48

Pantano C. 2004. Direct simulation of nonpremixed flame extinction in a methane-air jet with reduced chemistry. J. Fluid Mech. 514:231-70

Pantano C, Sarkar S, Williams FA. 2003. Mixing of a conserved scalar in a turbulent reacting shear layer. J. Fluid Mech. 481:291328

Pao YH. 1964. Statistical behavior of a turbulent multicomponent mixture with first-order reactions. AIAA J. 2:1550-59

Papavassiliou DV. 2002. Turbulent transport from continuous sources at the wall of a channel. Int. J. Heat Mass Transf. 45:3571-83

Papavassiliou DV, Hanratty TJ. 1997. Transport of a passive scalar in a turbulent channel flow. Int. J. Heat Mass Transf. 40:1301-11

Pasquero C, Provenzale A, Babiano A. 2001. Parameterization of dispersion in twodimensional turbulence. J. Fluid Mech. 439: 279-303

Peltier WR, Caulfield CP. 2003. Mixing efficiency in stratified shear flows. Annu. Rev. Fluid Mech. 35:135-67

Peters N. 1985. Numerical and asymptotic analysis of systematically reduced reaction schemes for hydrocarbon flames. Lect. Notes Phys. 241:90-109

Peters N. 2000. Turbulent Combustion. Cambridge, UK: Cambridge Univ. Press

Phillips WRC. 2001a. On the pseudomomentum and generalized Stokes drift in a spectrum of rotational waves. J. Fluid Mech. 430:209-29

Phillips WRC. 2001b. On an instability to Langmuir circulations and the role of Prandtl and Richardson numbers. J. Fluid Mech. 442:335-58

Phillips WRC. 2002. Langmuir circulations beneath growing or decaying surface waves. $J$. Fluid Mech. 469:317-42

Phillips WRC. 2004. On the spacing of Langmuir circulation in strong shear. J. Fluid Mech. Accepted
Pope S. 2000. Turbulent Flows. Cambridge, UK: Cambridge Univ. Press

Porté-Agel F, Meneveau C, Parlange MB. 2000. A scale-dependent dynamic model for largeeddy simulation: application to a neutral atmospheric boundary layer. J. Fluid Mech. 415:261-84

Pullin DI. 2000. A vortex-based model for the subgrid flux of a passive scalar. Phys. Fluids 12:2311-19

Pullin DI, Lundgren TS. 2001. Axial motion and scalar transport in stretched spiral vortices. Phys. Fluids 13:2553-63

Pullin DI, Saffman PG. 1994. Reynolds stresses and one-dimensional spectra for a vortex model of homogeneous anisotropic turbulence. Phys. Fluids 6:1787-96

Rayleigh. 1883. Investigation of the character of the equilibrium of an incompressible heavy fluid of variable density. Proc. London Math. Soc. 14:170-77

Richtmyer RD. 1960. Taylor instability in shock acceleration of compressible fluids. Commun. Pure Appl. Math. 13:297-319

Riley JJ, Lelong M-P. 2000. Fluid motions in the presence of strong stable stratification. Annu. Rev. Fluid Mech. 32:613-57

Robinson A. 1956. On the motion of small particles in a potential field of flow. Commun. Pure Appl. Math. 9:69-84

Saffman PG. 1960. On the effect of the molecular diffusivity in turbulent diffusion. J. Fluid Mech. 8:273-83

Sawford B. 2001. Turbulent relative dispersion. Annu. Rev. Fluid Mech. 33:289-317

Schneider T. 2004. The tropopause and the thermal stratification in the extratropics of a dry atmosphere. J. Atmos. Sci. 61:131740

Scotti A, Meneveau C. 1999. A fractal model for large-eddy simulation of turbulent flow. Phys. D 127:198-232

Shan JW, Dimotakis PE. 2001. Turbulent mixing in liquid-phase transverse jets. http:// resolver.library.caltech.edu/CaltechGalcitFM: 2001.006

Sharp DH. 1984. An overview of RayleighTaylor instability. Phys. D 12:3-18 
Shaw RA. 2003. Particle-turbulence interactions in atmospheric clouds. Annu. Rev. Fluid Mech. 35:183-227

Shaw RA, Reade WC, Collins LR, Verlinde J. 1998. Preferential concentration of cloud droplets by turbulence: effects on the early evolution of cumulus cloud droplet spectra. J. Atmos. Sci. 55:1965-76

Slessor MD. 1998. Aspects of turbulent-shearlayer dynamics and mixing. $\mathrm{PhD}$ thesis. Calif. Inst. Technol.

Slessor MD, Bond CL, Dimotakis PE. 1998. Turbulent shear-layer mixing at high Reynolds numbers: effects of inflow conditions. J. Fluid Mech. 376:115-38

Smith JA. 1992. Observed growth of Langmuir circulation. J. Geophys. Res. 97:565164

Smith JA. 1998. Evolution of Langmuir circulation in a storm. J. Geophys. Res. 103:1264968

Smith KS, Boccaletti G, Henning CC, Marinov I, Tam CY, et al. 2002. Turbulent diffusion in the geostrophic inverse cascade. J. Fluid Mech. 469:13-48

Sreenivasan KR. 1991. Fractals and multifractals in fluid turbulence. Annu. Rev. Fluid Mech. 23:539-600

Taylor GI. 1935. Statistical theory of turbulence: Parts I-II. Proc. Roy. Soc. London A 151:421-64

Taylor GI. 1950. The instability of liquid surfaces when accelerated in a direction perpendicular to their planes. Proc. R. Soc. London Ser. A 201:192-96

Taylor GI. 1953. Dispersion of soluble matter in solvent flowing slowly through a tube. Proc. R. Soc. London Ser. A 219:186-203

Taylor GI. 1954. The dispersion of matter in turbulent flow through a pipe. Proc. R. Soc. London Ser. A 223:446-68

Tennekes H, Lumley JL. 1972. A First Course in Turbulence. Cambridge, MA: MIT Press
Thorpe SA. 2004. Langmuir circulation. Annu. Rev. Fluid Mech. 36:55-79

Toor HL. 1962. Mass transfer in dilute turbulent and nonturbulent systems with rapid irreversible reactions and equal diffusivities. AIChE J. 8:70-78

Turner JS. 1973. Buoyancy Effects in Fluids. Cambridge, UK: Cambridge Univ. Press

Tziperman E. 1986. On the role of interior mixing and air-sea fluxes in determining the stratification and circulation of the oceans. J. Phys. Oceanogr. 16:680-93

Van Atta C, Antonia R. 1980. Reynolds number dependence of skewness and flatness factors of velocity gradients. Phys. Fluids 23:25257

Veron F, Melville WK. 2001. Experiments on the stability and transition of wind-driven water surfaces. J. Fluid Mech. 446:25-65

Vervisch L, Poinsot T. 1998. Direct numerical simulation of non-premixed turbulent flames. Annu. Rev. Fluid Mech. 30:655-91

Villermaux E. 2002. Mixing as an aggregation process. IUTAM Symp. Turbulent Mixing Combustion. 3-6 June 2001, pp. 1-21, Kingston, Ontario, Can.

Voelkl T, Pullin DI, Chan DC. 2000. A physical-space version of the stretchedvortex subgrid-stress model for large-eddy simulation. Phys. Fluids 12:1810-25

Wallace AK. 1981. Experimental investigation of the effects of chemical heat release in the reacting turbulent plane shear layer. $\mathrm{PhD}$ thesis. Univ. Adelaide

Warhaft Z. 2000. Passive scalars in turbulent flows. Annu. Rev. Fluid Mech. 32:203-40

Williams FA. 1985. Combustion Theory. Menlo Park, CA: Benjamin/Cummings

Wunsch C. 2002. What is the thermohaline circulation? Science 298:1179-81

Wunsch C, Ferrari R. 2004. Vertical mixing, energy, and the general circulation of the oceans. Annu. Rev. Fluid Mech. 36:281-314 
俩 Annual Review of Fluid Mechanics

Volume 37, 2005

\section{ConTENTS}

ROBERT T. JONES, ONE OF A KIND, Walter G. Vincenti 1

GeORge GABRIEL STOKES ON WATER WAVE THEORY, Alex D.D. Craik

Microcirculation AND HeMORHEOlOgy, Aleksander S. Popel and Paul C. Johnson

BLADEROW INTERACTIONS, TRANSITION, AND HIGH-LIFT AEROFOILS IN LOW-PRESSURE TURBINES, Howard P. Hodson and Robert J. Howell

The Physics of Tropical Cyclone Motion, Johnny C.L. Chan

Fluid MeChanics AND RHEOlOGY of DENSE SuSPENSIONS, Jonathan J. Stickel and Robert L. Powell

Feedback Control of Combustion Oscillations, Ann P. Dowling and Aimee S. Morgans

DisSECTING InSECT FLIGHT, Z. Jane Wang

Modeling Fluid Flow IN OIL ReSERVOIRS, Margot G. Gerritsen and Louis J. Durlofsky

IMMERSED BOUNDARY METHODS, Rajat Mittal and Gianluca Iaccarino

STRATOSPHERIC DYNAMICS, Peter Haynes

THE DYNAMICAL SYSTEMS APPROACH TO LAGRANGIAN TRANSPORT IN OCEANIC FLOWS, Stephen Wiggins

Turbulent MiXing, Paul E. Dimotakis

GLOBAL INSTABILITIES IN SPATIALly DEVEloping Flows:

NON-NORMALITY AND NONLINEARITY, Jean-Marc Chomaz

GRAVITY-DRIVEN BUBBLY FLOWS, Robert F. Mudde

PRINCIPLES OF MiCROFLUIDIC ACTUATION BY MODULATION OF

SuRfACE STRESSES, Anton A. Darhuber and Sandra M. Troian

Multiscale Flow Simulations Using Particles,

Petros Koumoutsakos 